\title{
Structural characterization of anti-inflammatory Immunoglobulin G Fc proteins
}

Alysia A. Ahmed ${ }^{1}$, John Giddens ${ }^{2}$, Andrew Pincetic ${ }^{3}$, Joseph V. Lomino², Jeffrey V. Ravetch ${ }^{3}$, Lai-Xi Wang ${ }^{2}$, Pamela J. Bjorkman ${ }^{1,4,5}$ 


\begin{abstract}
Immunoglobulin $\mathrm{G}(\mathrm{lg} G)$ is a central mediator of host defense due to its ability to recognize and eliminate pathogens. The recognition and effector responses are encoded on distinct regions of IgGs. The diversity of the antigen recognition Fab domains accounts for IgG's ability to bind with high specificity to essentially any antigen. Recent studies have indicated that the Fc effector domain also displays considerable heterogeneity, accounting for its complex effector functions of inflammation, modulation and immune suppression. Therapeutic anti-tumor antibodies, for example, require the pro-inflammatory properties of the IgG Fc to eliminate tumor cells, while the antiinflammatory activity of Intravenous Immunoglobulin G (IVIG) requires specific Fc glycans for activity. In particular, the anti-inflammatory activity of IVIG is ascribed to a small population of IgGs in which the Asn297-linked complex $N$-glycans attached to each $\mathrm{Fc}_{\mathrm{H}} 2$ domain include terminal $\alpha 2,6$-linked sialic acids. We used chemoenzymatic glycoengineering to prepare fully di-sialylated IgG Fc and solved its crystal structure. Comparison of the structures of asialylated Fc, sialylated Fc, and F241A Fc, a mutant that displays increased glycan sialylation, suggests that increased conformational flexibility of the $\mathrm{C}_{\mathrm{H}} 2$ domain is associated with the switch from pro- to anti-inflammatory activity of the Fc.
\end{abstract}

\title{
KEYWORDS
}

sialylated IgG Fc, inflammation, IVIG, N-linked glycan, X-ray crystallography 


\section{INTRODUCTION}

IVIG is used worldwide as an antibody replacement therapy and as a treatment for a variety of inflammatory disorders [1]. IVIG is pooled IgG from the serum of healthy donors. It contains the four different subtypes of human $\lg G(\lg G 1, \lg G 2, \lg G 3$, and IgG4) and over 30 glycovariants [2]. Although the beneficial effects of IVIG as an antiinflammatory preparation are apparent, the mechanism of action has only recently begun to be understood as being correlated with the relatively constant portion, or Fc region, of IgGs [3]. The Fc region of IgG interacts with a variety of receptors, determined by the amino acid and glycan composition of the Fc. Among these receptors are the canonical

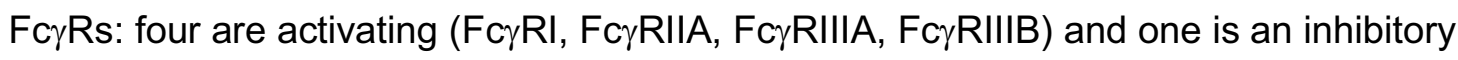
receptor (FcyRIIB) [3]. The simultaneous expression of these receptors on effector cells sets a threshold for inflammatory responses [4]. IVIG causes an up-regulation of the inhibitory receptor Fc $\gamma$ RIIB, initiating anti-inflammatory responses [5].

A downside of IVIG therapy is that it is only effective when administered at high doses $(1-3 \mathrm{~g} / \mathrm{kg})$ when treating inflammatory diseases [6]. Rationalizing the high dose requirements, the anti-inflammatory activity of IVIG has been attributed to a small population of IgGs with $\alpha 2,6$-sialyated $N$-linked glycans attached to a conserved asparagine (Asn297) in the $\mathrm{Fc} \mathrm{C}_{\mathrm{H}} 2$ domain [7]. The Asn297-linked carbohydrate is a complex, usually core-fucosylated, biantennary glycan containing a pentasaccharide $\mathrm{Man}_{3} \mathrm{GlcNAc}_{2}$ core (Man, mannose; GlcNAc, N-acetylglucosamine), which can be modified by addition of terminal galactose or sialic acid [8] (Fig. 1). Approximately 10\% of the Fc regions in IVIG terminate in sialic acid, of which $1-3 \%$ are disialylated $[2 ; 9 ; 10]$. In vivo studies have shown that Fc sialylation is the main determinant in IVIG antiinflammatory activity $[9 ; 11]$. For example, IVIG that was enriched in terminally-sialylated Fc glycans showed a 10-fold increase in anti-inflammatory activity [7; 12], and if IVIG was treated with neuraminidase to remove terminal sialic acids or with PNGase F to 
remove the entire $N$-linked glycan, the anti-inflammatory activity of IVIG was abrogated [9]. Similar results were found for recombinant, 2,6 sialylated IgG1 [7]. In mouse models, including a serum-induced arthritis model in mice, sialylated Fc (sFc; defined here as referring to a mixture of sialylated glycoforms) decreased inflammation of ankle joints [9], and IVIG activity was shown to be dependent upon terminally-sialylated Fc regions in four independent in vivo model systems of autoimmune disease mediated by autoantibodies [11].

The anti-inflammatory properties of sFc have been attributed to the effect of sialylation switching the specificity of Fc binding to its receptors. In vivo experiments showed that the anti-inflammatory effects of sFc required expression of the C-type lectinlike receptor specific intracellular adhesion molecule-grabbing non-integrin R1 (SIGN-RI) (the mouse homolog of human dendritic cell-specific intercellular adhesion molecule-3grabbing nonintegrin; DC-SIGN), leading to a model in which conformational changes in Fc resulting from sialylation of the Asn297-attached glycan allowed interactions with members of the SIGN receptor family [13]. Indeed, previous structural studies demonstrated that modification of the Asp297-linked glycan can affect Fc structure. For example, it was shown that the glycan contributed to an "open" conformation of IgG Fcs, in which the $\mathrm{C}_{\mathrm{H}} 2$ domains were separated by a larger distance than in deglycosylated Fc structures [14]. If the IgG Fc glycan was enzymatically removed, the $\mathrm{C}_{\mathrm{H}} 2$ region adopted a "closed" state [15]. Conformational changes have also been observed when individual sugar residues on the Fc-linked glycan were modified. When fucose was removed, a subtle change involving Tyr296 was observed in X-ray crystallographic and NMR structures [16; 17]. This modification resulted in an increased affinity for the activating receptor FcyRIIIa, leading to enhanced antibody-dependent cellular cytotoxicity (ADCC) activity [18; 19]. Solution NMR studies have reported increased mobility of the glycan 
arms upon sialylation, further supporting the contention that alterations in the glycan composition can influence the structure of the Fc [20].

Here we solved the crystal structure of a chemically-homogeneous disialylated Fc (di-sFc) and compared it to new structures of a partially sialylated Fc (F241A Fc) and wtFc, as well as to wtFc and glycomutant Fc structures available in the protein data bank (PDB). We found that di-sFc and F241A Fc show increased conformational heterogeneity in crystals compared to wtFc, a characteristic that may relate to sialylation and anti-inflammatory properties.

\section{RESULTS}

\section{Glycan analysis of purified Fc proteins}

Proteins were produced by transient transfection in HEK 293-6E cells as IgG1 Fc fragments (wtFc, F241A Fc and F243A Fc) or in a stably-transfected Chinese hamster ovary $(\mathrm{CHO})$ cell line (wtFc) [21]. Disialylated sFc (di-sFc) was prepared by chemoenzymatic glycoengineering [22] of an Fc fragment isolated after papain cleavage of Rituximab, a human IgG1.

Carbohydrate analyses of $\mathrm{N}$-glycans released from the Fc proteins revealed that the $\mathrm{N}$-glycans attached to Asn297 of wtFc expressed in either HEK293 or CHO cells consisted of only three glycoforms: G0F, G1F, and G2F (Fig. 2a,b,). This result was consistent with previous studies investigating glycoforms of IgGs expressed in HEK cells [23]. Neither mono- nor disialylated $N$-glycans on either form of wtFc were detected. By contrast, the glycans attached to the F241A and F243A Fc mutants contained more diverse glycoforms: in addition to the G0F, G1F, and G2F glycoforms, significant portions of the $N$-glycans carried a bisecting GlcNAc moiety and contained terminal sialic acids (Fig. 2c,d,). The sialylated forms included about $20 \%$ of terminally monosialylated $N$-glycans (S1G2F and other monosialylated glycoforms) and about $4 \%$ of disialylated $N$ - 
glycans (S2G2F and other disialylated glycoforms). These results were consistent with a recent study of the F241A and F243A Fc mutants [23], suggesting that these substitutions promote further processing of Fc glycans resulting in generation of sialylated and bisecting GIcNAc-containing glycoforms. The chemoenzymatically remodeled Fc (di-sFc) was almost homogeneous: $>95 \%$ of the $N$-glycans were the disialylated glycoform (Fig. 2e, Fig. S1).

We also compared the Fc proteins in a Western blot using Sambucus Nigra (SNA), a lectin that binds preferentially to $\alpha 2,6$-linked sialic acid attached to a terminal galactose [24]. As expected, SNA blots of wtFc, F241A, F243A and di-sFc proteins demonstrated sialylation of di-sFc, F241A Fc and F243A Fc, but not wtFc (Fig. 3). These results were consistent with previous reports of partial sialylation of the $\mathrm{N}$-glycans of F241A and F243A Fc [25].

Liquid chromatography/electrospray ionization mass spectrometry (LC-ESI-MS) was used to analyze wtFc that was expressed as an Fc fragment in HEK cells before and after complete removal of the $N$-glycans by PNGase F treatment. The analysis revealed two major species that were 950 and 1315 Da larger than the calculated molecular mass of the polypeptide backbone following PNGase F treatment, suggesting the existence of additional posttranslational modifications (Fig. S2a). A similar analysis of wtFc expressed in $\mathrm{CHO}$ cells revealed three major species that were 269,922 , and $1218 \mathrm{Da}$ larger than the calculated molecular mass of the polypeptide backbone (Fig. S2b). The results for the F241A Fc and F243A Fc proteins that were expressed in HEK cells were the same as the HEK-expressed wtFc (Fig. S2c,d). In contrast, an Fc fragment prepared from papain cleavage of an intact IgG1 (Rituximab) did not contain posttranslational modifications other than the $\mathrm{N}$-glycans (Fig. S2e). A monosaccharide composition analysis on the recombinant Fcs after removal of the $\mathrm{N}$-glycans with PNGase F revealed the presence of GalNAc, GlcNAc, Gal, and Neu5Ac (Fig. S3b,c), 
suggesting the existence of O-linked glycans. In contrast, the Fc fragment prepared from papain cleavage of Rituximab did not contain any monosaccharides after PNGase F cleavage (Fig. S3d). On the basis of the additional molecular mass (950 Da) in comparison with the polypeptide backbone, the major O-glycan on the HEK-expressed Fcs appeared to be a core 1 structure carrying two sialic acid residues [calculated mass for $(\mathrm{Neu} 5 \mathrm{Ac})_{2}-\mathrm{Gal}-\mathrm{GalNAc}-\mathrm{H}_{2} \mathrm{O}=947.86 \mathrm{Da}$. In the $\mathrm{CHO}$-expressed wtFc, an additional mass of $1218 \mathrm{Da}$ on the polypeptide backbone was observed. However, this additional mass does not correspond to typical O-glycan structures, implicating potential additional modifications on the core structures; e.g., acetylation on the sialic acid moieties. The detailed structures of the O-glycans remain to be determined. However, the lack of reactivity of HEK-expressed wtFc in the SNA blot (Fig. 3) demonstrated that there were no $\alpha 2,6$-linked sialic acids on the putative O-glycans. Since the hinge region of IgG where the O-glycans would likely be located is usually disordered in Fc crystal structures, these glycans (if present) would not be visible in published structures of recombinantly-expressed Fc fragments [23; 26; 27; 28]. Nor are there apparent differences in the structural properties of Fcs that correlate with whether they were expressed as Fc fragments versus prepared by cleavage of intact lgG (Table 1).

The finding of putative O-glycans on proteins expressed as Fc fragments, but not on an Fc fragment derived by cleavage of intact lgG, could be explained if expression of Fc as a fragment allows access during biosynthesis by O-glycan-adding enzymes in the Golgi apparatus to a region of the $\lg G$ hinge that is inaccessible when intact lgG is trafficking through the Golgi. Although there were no apparent structural differences in Fcs as a function of whether or not they were produced in a form predicted to contain the putative O-glycan, these results suggest that functional studies employing Fcs expressed directly as fragments should compare results with Fcs derived by enzymatic cleavage of intact lgGs to rule out potential effects of unnatural O-glycosylation. 


\section{wtFc structures are relatively similar}

In order to assess potential conformational differences in Fc structures, we first compared wtFc structures available in the Protein Data Bank (PDB) and a $2.4 \AA$ resolution wtFc structure solved in this study (space group P $2{ }_{1} 2{ }_{1}{ }_{1}$; one molecule per asymmetric unit) (Supplementary Table 1). We defined "wild type" as those Fc structures that were derived from lgGs or Fc fragments containing the naturally-occurring mixture of $N$-glycans attached to Asn297 when the protein was produced in mammalian cells, which included our new wtFc structure and PDB entries 1H3X, 3AVE, 2DTS, 3DO3, $1 \mathrm{FC} 1$ and $1 \mathrm{HZH}$. Fc fragments are homodimers in which the $\mathrm{N}$-terminal $\mathrm{C}_{\mathrm{H}} 2$ domains contact each other through their Asn297-linked $\mathrm{N}$-glycans, and the C-terminal $\mathrm{C}_{\mathrm{H}} 3$ domains form a stable interface that is relatively constant between structures (Fig. 1b and 4a). Although the $\mathrm{C}_{\mathrm{H}} 2$ domain $\mathrm{N}$-glycans are generally heterogeneous in wtFc proteins (Fig. 2), ordered electron density is usually observed for eight or nine carbohydrate residues of the biantennary complex glycan attached to Asn297 of each $\mathrm{C}_{\mathrm{H}} 2$ domain (PDB codes: 1H3X, 3AVE, 2DTS, 3DO3, 1FC1 and 1HZH).

In order to evaluate potential differences in wtFc and sialylated Fc structures (Fig. 4), the $\mathrm{C}_{\mathrm{H}} 3$ domains of the wtFc structures were aligned, and we monitored the separation of the $\mathrm{C}_{\mathrm{H}} 2$ domains as previously described [29] by measuring distances between the $\mathrm{C} \alpha$ atoms at $\mathrm{C}_{\mathrm{H}} 2$ positions $238,241,301$, and 329 . The differences in $\mathrm{C}_{\mathrm{H}} 2$ domain separation (Table 1) relative to a published wtFc structure (3AVE) were plotted for each set of paired C $\alpha$ positions (Fig. 5). Despite differences in crystal packing, the wtFc structures exhibited only small deviations in $\mathrm{C}_{\mathrm{H}} 2$ domain separation distances, demonstrating relatively little structural variability. By contrast, a low-resolution wtFc structure solved from crystals grown under high salt conditions [14] (PDB: 1H3Y) showed deviations from the other wtFc structures that were crystallized in lower salt 
concentrations (Fig. 5, Table 1). Structures of Fc proteins with different defined glycoforms showed a larger degree of variability in $\mathrm{C}_{\mathrm{H}} 2$ domain separation than wtFc structures, as was also true for Fc structures with mutations introduced to enhance effector functions, and Fc structures solved as complexes with other proteins (Fig. 5). There were no systematic differences in $\mathrm{C}_{\mathrm{H}} 2$ domain separation or structure based on whether or not the Fc fragment was predicted to contain an O-glycan in its hinge region (Table 1; Fig. 5).

\section{Structures of two forms of di-sFc}

A 3.0 A resolution of di-sFc was solved in space group $\mathrm{P} 2{ }_{1}$ with two di-sFc molecules per asymmetric unit. The structure was compared with wtFc structures and a previously described structure of sFc that included a mixture of di- and monosialylated Fc (PDB: 4BYH) [30]. In the di-sFc structure, electron density for biantennary $N$-linked glycans was visible for all four di-sFc chains in the two di-sFc dimers, but although the di-sFc protein was fully disialylated (Fig. 2e), electron density for a terminal sialic acid was found only on the 6 -arm of chains $B$ and $C$, with the 6 -arm of chains $A$ and $D$ showing ordered glycan residues up to a GICNAc or galactose, respectively, and the 3-arm showing electron density for glycan moieties up to the GlcNAc (chains $A, B, C$ and D) (Fig. 6). Similarly, sialic acid was visible only on the 6-arm of chain A in the 4BYH sFc structure [30]. In all three sFc structures (in 4BYH as well as in the two copies of di-sFc in the new sFc structure), the 6- and 3-arms of the biantennary glycans projected away from the protein surface and were solvent exposed; thus the ordered sialic acid on the glycan 6-arm was not positioned into a pocket formed by Glu258 and Lys290, as previously proposed [13].

Interestingly, the two di-sFc dimers in the asymmetric unit of the $\mathrm{P} 2{ }_{1}$ crystals exhibited differences in the arrangement of their $\mathrm{C}_{\mathrm{H}} 2$ domains. One di-sFc (chains $\mathrm{A}$ and 
B) exhibited a relatively "closed" conformation, as defined by the separation between the two $\mathrm{C}_{\mathrm{H}} 2$ domains, whereas the second di-sFc (chains $\mathrm{C}$ and $\mathrm{D}$ ) exhibited a relatively "open" conformation (Table 1 and Fig. 4b). As compared with a representative wtFc structure (3AVE), the open di-sFc exhibited a $6.8 \AA$ root mean square deviation (RMSD) (calculated for the $103 \mathrm{C} \alpha$ atoms of one $\mathrm{C}_{\mathrm{H}} 2$ domain after alignment of the partner $\mathrm{C}_{\mathrm{H}} 2$ domain in each structure), as compared a $15.0 \AA$ RMSD between the wtFc and closed di-sFc $\mathrm{C}_{\mathrm{H}} 2$ domains, a $7.9 \AA$ RMSD between the open di-sFc and the $4 \mathrm{BYH}$ sFc, and a 8.8 $\AA$ RMSD between the closed di-sFc and the $4 \mathrm{BYH} \mathrm{sFc}$. To quantitatively evaluate $\mathrm{C}_{\mathrm{H}} 2-\mathrm{C}_{\mathrm{H}} 2$ domain separation distances, which cannot be easily defined by a single number reflecting an average separation, we used a previously-defined metric in which the distance between four partner $\mathrm{C} \alpha$ atoms on each $\mathrm{C}_{\mathrm{H}} 2$ domain are measured (Pro238, Pro329, Phe241 and Arg301) and compared between structures [29]. In addition to determining $\mathrm{C}_{\mathrm{H}} 2-\mathrm{C}_{\mathrm{H}} 2$ separation distances for these $\mathrm{C} \alpha$ residues (Table 1), we calculated the differences relative to a reference wtFc structure (3AVE) to determine how greatly the various structures deviated from wtFc and other Fc structures. As defined by the distances between these $\mathrm{C} \alpha$ atoms, the closed di-sFc exhibited a greater difference in $\mathrm{C}_{\mathrm{H}} 2$ domain separation when compared to wtFc structures than $4 \mathrm{BHY} \mathrm{sFc}$, whereas the open di-sFc $\mathrm{C} \alpha$ exhibited a difference within the range observed for other wtFc structures (Fig. 5).

We next aligned individual $\mathrm{C}_{\mathrm{H}} 2$ domains of the closed di-sFc, open di-sFc, wtFc ( $3 \mathrm{AVE}$ ) and the $4 \mathrm{BYH}$ sFc structures to compare the orientations of hydrophobic side chains (Phe241 and Phe243) adjacent to the N-linked glycan attached to Asn297 (Fig. 7a). No major changes were observed in the overall fold of the $\mathrm{C}_{\mathrm{H}} 2$ domains, and only minor changes were found in the Phe243 side chain orientation. However, larger changes were observed in the Phe 241 side chain, which was rotated $\sim 90^{\circ}$ compared to Phe241 in other Fc structures (Fig. 7b). Typically the aromatic rings of the Phe241 and 
Phe243 side chains were stacked with respect to each other; however in both $\mathrm{C}_{\mathrm{H}} 2$ domains of the closed and open di-sFc structures, Phe241 adopted a different conformation such that it was no longer stacked with Phe243 (Fig. 7b,c and Fig. S4). The Phe241 side chain was not involved in crystal contacts in any of the structures (data not shown); thus these differences were not related to crystal packing. Although the Asn297-linked N-glycan is adjacent to Phe241 and Phe243, no major differences were observed in the glycan conformation (Fig. 7a), thus the Phe241 side chain orientation did not affect the glycan conformation.

\section{Structure of F241A Fc}

We also solved a 2.2 Å resolution crystal structure of F241A Fc (space group P $2{ }_{1} 2_{1} 2_{1}$; one molecule per asymmetric unit) and compared it to wtFc and sFc structures and to PDB code 4BM7, a previous F241A Fc structure [23] (Fig. 8a, b). On chain A of the new F241A Fc structure, there was ordered electron density for residues Pro230 to Leu443, which included part of the hinge region (Glu216 to Pro230 for human IgG1 proteins) that normally links the Fab to the N-terminus of the Fc region (Fig. 8c). By contrast, the first ordered residue on both chains of wtFc structures was usually Leu234, Leu235, Gly236, Gly237, or Pro238 (PDB codes: 1H3X, 3AVE, 2DTS, 3DO3, 1FC1 and 1HZH), and the first ordered residue of the F241A B chain was Gly237. The hinge region was also disordered in the 4BM7 F241A Fc structure (the first ordered residues were Gly237 on chain A and Pro238 on chain B). At Asn297, six ordered glycan residues were modeled on chain A and nine residues on chain B of the new F241A structure (Fig. 6), but sialic acids were not visible in the new F241A or the 4BM7 F241A structures although the F241A glycans included both mono- and disialylated species [23] (Fig. 2 and Fig. 8b). Because $N$-glycans were not completely resolved even in the di-sFc structure, which was solved from crystals containing Fc protein with completely homogeneous $N$-glycans, 
we cannot use the F241A Fc structures to determine the compositions of $N$-glycans in the F241A Fc crystals. Therefore it is not possible to determine whether a defined glycan species crystallized from the heterogeneous protein preparations used to produce both of the F241A Fc structures and all of the wtFc structures.

Comparison of the $\mathrm{C}_{\mathrm{H}} 2$ domain separations of the F241A Fc structure with other Fc structures showed that the new F241A Fc structure was similar to wtFc structures and the open di-sFc structure, but substantially more closed than the 4BM7 F241A Fc (Fig. 5, Table 1). (Because portions of the chain B $\mathrm{C}_{\mathrm{H}} 2$ domain of the 4BM7 F241A Fc structure were disordered, including the Asn297-linked glycan and residues 264-274 (BC loop), 296-300 (C'E loop), and 324-332 (FG loop), we could not measure all of the interchain distances in the 4BM7 F241A Fc structure.) This large degree of difference in F241A Fc conformations is similar to that observed for di-sFc conformations but distinct from wtFc conformations. Since both of the F241A Fc structures (the structure reported here and the 4BM7 structure) were derived from proteins that were partially sialylated, this observation is consistent with the suggestion that a greater degree of conformational heterogeneity is available to sialylated Fc proteins than to wtFc with conventional glycosylation species. Alternatively, or perhaps in addition, the F241A substitution itself could result in greater conformational heterogeneity.

\section{DISCUSSION}

The Fc regions of IgGs can function to either promote or suppress the inflammatory response. Previous studies have shown that the Asn297-linked N-glycan composition is a determining factor in function [12; 31]. Both biochemical and in vivo studies have shown that sialylation of the Fc glycan is essential for the anti-inflammatory activity of IVIG [2; 9; 12]. Here we compared structures of wtFc, which carries mostly asialylated $\mathrm{N}$-glycans, to sialylated forms of $\mathrm{Fc}$, both a fully disialylated form produced by 
chemoenzymatic glycoengineering, and an F241A mutant form of Fc. Compared with wtFc, the Asn297-linked N-glycan of F241A and F243A Fc showed increased mono- and disialylated products when the protein was expressed in mammalian cells. This suggests that IgGs with an F241A mutation may be effective anti-inflammatory molecules due to increased sialylation.

Our analyses of the wtFc proteins produced in two different mammalian cell lines demonstrated that the Asn297-linked N-glycans were mainly G0F, G1F and G2F glycoforms, consistent with previous studies [23], thus we assume a similar composition for the $\mathrm{N}$-glycans on the relatively structurally-invariant wtFc structures in the pdb. Not only are these glycans mostly asialylated, they also contain only a small percentage of digalactosylated forms. In a systematic comparison of wtFc structures reported here and previously, we found only small deviations in the "openness" of the Fc, evaluated by the distance between the partner $\mathrm{C}_{\mathrm{H}} 2$ domains in the $\mathrm{Fc}$ dimer, despite different crystal packing environments, however, we noted that certain glycan species cause increased variation in crystal structures. For example, the F241A Fc structure reported here and one described previously [23] showed a larger degree of variation in $\mathrm{C}_{\mathrm{H}} 2$ domain orientations than seen in a survey of seven wtFc structures. Other glycovariants of Fc, including a recent structure of a digalactosylated form of wtFc [32], also showed a larger degree of conformational variability as assessed by differential $\mathrm{C}_{\mathrm{H}} 2$ domain separations.

Similar to the partially sialylated F241A Fc structures, crystal structures of sialylated wtFc (sFc) have been found in several conformations. In our crystal structure of fully disialylated $\mathrm{Fc}$, we found both open and closed $\mathrm{C}_{\mathrm{H}} 2$ domain conformations in the same crystal, and a previously published sFc structure [30] revealed a conformation similar to the canonical wtFc structures, again suggesting a large degree of inter-domain flexibility of sialylated forms of Fc compared to the relatively invariant structures of asialylated wtFc. 
Recent molecular dynamics simulations showed a wide range of conformations that Fc can adopt [32]. Here we have documented a greater flexibility of glycovariant forms of Fc that may be key to the ability of the Fc region of IgG to modulate inflammatory and anti-inflammatory activities mediated by glycan differences. Further structural investigations of how glycan composition and conformational flexibility affect binding to Fc receptors that mediate differential activities will be critical to understanding the significance of these results. 


\section{Figure legends}

Fig. 1. IgG, Fc and glycan structures. (a) Schematic structure of IgG1. (b) Ribbon diagram of wtFc (PDB: 3AVE) with Asn297-linked N-glycans shown as sticks. Glycan residues are colored as in panel c. (c) Schematic of an Asn297-linked N-glycan. The biantennary glycan consists of a mannose (Man) and $\mathrm{N}$-acetylglucosamine (GlcNAc) core that can be modified by addition of core fucosylation (Fuc), a bisecting GlcNAc, terminal galactose (Gal) and sialic acid (NeuNAc).

Fig. 2. HPAEC-PAD analysis of PNGase F-released N-glycans of IgG Fc proteins. (a) wtFc expressed in HEK cells. (b) wtFc expressed in CHO cells. (c) F241A Fc expressed in HEK cells. (d) F243A expressed in HEK cells. (e) di-sFc prepared by chemoenzymatic glycoengineering of an Fc fragment isolated after papain cleavage of Rituximab. The amounts of mono- and disialylated $\mathrm{N}$-glycans are shown. * indicates a mixture of heterogeneous monosialylated glycoforms. † indicates a mixture of heterogeneous disialylated glycoforms. $\ddagger$ indicates a satellite peak due to epimerization of reducing end GlcNAc to ManNAc.

Fig. 3. SDS-PAGE (left) and SNA blot (right) of Fc proteins expressed in HEK cells. wtFc, F241A Fc and F243A Fc were expressed as Fc fragments; di-sFc was prepared by papain cleavage from Rituximab lgG.

Fig. 4. Comparison of wtFc and sFc structures. Fc structures were aligned by superimposing the $\mathrm{C}_{\mathrm{H}} 3$ domains. (a) Alignment of wtFc structures (the wtFc structure solved in this study plus PDB entries $1 \mathrm{HZH}, 1 \mathrm{FC} 1,2 \mathrm{DTS}, 3 \mathrm{AVE}, 1 \mathrm{H} 3 \mathrm{X}$, and 3DO3). (b) Alignment of closed and open structures of di-sFc (this study), sFc (PDB 4BYH), and wtFc (PDB 3AVE). 
Fig. 5. Comparison of $\mathrm{C}_{\mathrm{H}} 2$ domain separation in Fc structures. Left: location of $\mathrm{C} \alpha$ atoms for Pro238, Phe241, Arg301, and Pro329 (red spheres) and Asn297 (blue spheres) indicated on the $\mathrm{C}_{\mathrm{H}} 2$ domains of wtFc (PDB: 3AVE). $\mathrm{C}_{\mathrm{H}} 2$ domain separation in individual Fc structures was evaluated by measuring distances (dotted lines) between the corresponding red spheres on each chain. Right: Differences in $\mathrm{C}_{\mathrm{H}} 2$ domain separation relative to a reference wtFc structure (3AVE) plotted for the each set of paired $\mathrm{C} \alpha$ positions in the indicated category of Fc structure. When a structure category had more than one member, the mean and standard deviation are superimposed upon the values. Fc structure categories are as follows: wtFc (structure solved in this study plus PDB entries 1H3X, 3AVE, 2DTS, 3DO3, 1FC1, 1HZH), wtFc high salt (1H3Y), glycovariant (GV) Fcs (1H3W, 1H3U, 1H3V, 2WAH, 4ACP, 3DNK,4KU1 and 3S7G), mutant (mut) Fcs (2QL1, 3FJT, and 3C2S), Fcs complexed with other proteins (com Fc) (3D6G, 1L6X, 1OQO, 1OQX, 1DN2, 1FCC, 1FC2, 1E4K, 1T83, 1T89, 3AY4, 3RY6, 3SGK and 3SGJ), di-sFc closed, di-sFc open, sFc (4BYH), F241A Fc (this study), and F241A Fc (4BM7).

Fig. 6. Comparison of ordered glycans on Fc structures. 2Fo-Fc electron density (contoured at $0.7 \sigma$ ) from an annealed omit map in the region of the Asn297-linked $\mathrm{N}$ glycan is shown for both chains of di-sFc closed, di-sFc open, F241A Fc, and wtFc (this study). Ordered glycan residues are shown schematically below each map using the colors and shapes defined in Fig. 1c.

Fig. 7. Comparison of glycan and protein residue conformations in sFc and wtFc structures. (a) Alignment of Asn297-linked $N$-glycans of di-sFc closed (blue), di-sFc open (green), F241A Fc (magenta), F241A Fc 4BM7 (yellow), sFc 4BYH (cyan), wtFc from this study and wtFc 3AVE (both gray). The side chains of Phe241 and Phe243 are 
shown in red on the $\mathrm{C}_{\mathrm{H}} 2$ domain of wtFc. (b) Alignment of Phe(Ala)241-Leu242-Phe243 region in the structures of di-sFc closed (blue), di-sFc open (green), F241A Fc (magenta), F241A Fc 4BM7 (yellow), sFc 4BYH (cyan), wtFc from this study and wtFc 3AVE (both gray).(c) 2Fo-Fc electron density (contoured at $0.7 \sigma$ ) from an annealed omit map in the region Phe243 and Phe241 side chains for the di-sFc structures. See also Fig. S5.

Fig. 8. Comparison of F241A Fc structures. (a) Superimposition of $\mathrm{C} \alpha$ traces of F241A Fc (this study) (magenta) and F241A Fc (PDB 4BM7) (cyan). Locations of $\mathrm{C} \alpha$ atoms at position 241 of each chain are shown as green spheres. (b) 2Fo-Fc electron density (contoured at $0.7 \sigma$ ) from annealed omit maps for F241A Fc (this study) and F241A Fc (PDB: 4BM7). Ordered glycan residues are shown schematically below each map using the colors and shapes defined in Fig. 1c. (c) 2Fo-Fc electron density (annealed omit map) of an ordered portion of the hinge region (blue) on chain A contoured at $0.6 \sigma$. (d) 2Fo-Fc electron density (contoured at $0.7 \sigma$ ) from annealed omit maps in the region of the Phe243 and Ala241 side chains for the structures of F241A Fc (this study) (left) and F241A Fc (PDB: 4BM7) (right).

Fig. S1. LC-ESI-MS analysis of reduced Rituximab Fc. (a) Reduced Rituximab Fc treated with wild type EndoS (calculated MW = 25289 Da). (b) Reduced di-sFc produced from Rituximab Fc (transglycosylated product) treated with wild type EndoS (calculated $\mathrm{MW}=27288 \mathrm{Da})$

Fig. S2. LC-ESI-MS analysis of reduced recombinant Fc fragments before (left panels) and after (right panels) PNGase F treatment to completely remove $\mathrm{N}$-glycans. ESI-MS spectra after deconvolution are shown for (a) wtFc (HEK cells) [calculated MW after 
PNGase F treatment $=25838 \mathrm{Da}$; experimentally found MW = 26788 and $27153 \mathrm{Da}$ (+950 and +1315 Da, respectively)]; (b) wtFc (CHO cells) [calculated MW after PNGase $\mathrm{F}$ treatment $=25337 \mathrm{Da}$; experimentally found $\mathrm{MW}=25606,26259$, and $26555 \mathrm{Da}$ (+269, +922, and +1218 Da, respectively)]; (c) F241A Fc (HEK cells) [calculated MW after PNGase F treatment = 25762 Da; experimentally found MW = 26713 and $27077 \mathrm{Da}$ (+951 and +1315 Da, respectively)]; (d) F243A Fc (HEK cells) [calculated MW after PNGase F treatment $=25762 \mathrm{Da}$; experimentally found MW = 26713 and $27077 \mathrm{Da}$ (+951 and +1315 Da, respectively)]; (e) wtFc fragment prepared by papain digestion of Rituximab lgG [calculated MW after PNGase F treatment = 24938 Da; experimentally found MW = $24938 \mathrm{Da}$.

Fig. S3. HPAEC-PAD neutral monosaccharide compositional analyses of putative Oglycans. Left panels: neutral monosaccharide compositional analyses. Right panels: sialic acid compositional analyses. (a) Monosaccharide standards. (b) Analytes from wtFc expressed in HEK cells after complete removal of $\mathrm{N}$-glycans. (c) Analytes from wtFc expressed in $\mathrm{CHO}$ cells after complete removal of $\mathrm{N}$-glycans. (d) Analytes from Fc fragment isolated after papain digest of Rituximab.

Fig. S4. Comparison of glycan and protein residue conformations in sFc and wtFc structures (see also Fig. 7). (a) Glycan conformation in the vicinity of the Phe241 and Phe243 residues (highlighted in red). Glycan residues are colored as in Fig. 1c. (b) Schematic structures of ordered glycan residues using the colors and shapes defined in Fig. 1c. (c) 2Fo-Fc electron density (contoured at $0.7 \sigma$ ) from annealed omit maps in the region of the Phe243 and Phe241 side chains for the indicated structures. 


\section{METHODS}

\section{Protein Expression and Purification}

Fc proteins were expressed in transiently-transfected HEK 293-6E suspension cells (F241A Fc and F243A Fc) or stably-transfected Chinese hamster ovary cells (wtFc) as described [21;33]. Fc proteins were isolated from harvested supernatants using protein A chromatography (GE Healthcare) followed by size exclusion chromatography in $25 \mathrm{mM}$ Tris-Cl pH 7.5, $100 \mathrm{mM} \mathrm{NaCl}$ on a Superdex 16/60 gel-filtration column (GE Healthcare). Fractions corresponding to the Fc were pooled and used for crystallization and glycan analysis.

Fc protein for di-sFc production was prepared by papain cleavage of Rituximab (Rituxan®) (Genentech, Inc., California), a human IgG1 Fc. Rituximab (40 mg) was buffer exchanged into $10 \mathrm{mM}$ Tris- $\mathrm{HCl} \mathrm{pH} 7.5$ overnight at $4^{\circ} \mathrm{C}$. Dialyzed material was concentrated to $20 \mathrm{mg} / \mathrm{mL}$ ( 2 mL). Immobilized papain (Thermo Scientific) was prepared following the manufacturer's protocol. $1 \mathrm{~mL}$ of immobilized papain slurry $(50 \%$ immobilized papain) was added to the Rituximab solution. The reaction was incubated at $37^{\circ} \mathrm{C}$ for 5 hours while continuously inverting the sample tube. The resin was removed using a spin column and the filtrate was loaded over a protein A column $(2 \times 5 \mathrm{~mL})$ that was pre-equilibrated with $10 \mathrm{mM}$ Tris- $\mathrm{HCl}, \mathrm{pH}$ 7.5. The column was washed with 10 column volumes of $10 \mathrm{mM}$ Tris- $\mathrm{HCl}, \mathrm{pH} 7.5$ until no protein was detected in the flow through. Bound $\mathrm{Fc}$ was eluted in $4 \mathrm{~mL}$ of a $100 \mathrm{mM}$ citric acid solution $\mathrm{pH} 3.5$. Eluted fractions were quickly neutralized using a $1.5 \mathrm{M}$ solution of $\mathrm{Tris}-\mathrm{HCl} \mathrm{pH} 8.8$. Purified $\mathrm{FC}$ was combined, concentrated and injected over a $40 \mathrm{~mL}(4 \times 10 \mathrm{~mL})$ HiTrap desalting column (GE Healthcare) pre-equilibrated in a phosphate buffered saline solution ( $\mathrm{pH} 7.4$ ) to give $10 \mathrm{mg}$ of Fc. wtFc for crystallization was prepared similarly by papain digestion of the anti-HIV-1 IgG1 antibody 21c (expressed in HEK 293-6E cells as described [33]). 


\section{Preparation of disialylated Fc (di-sFc)}

Purified Fc (10 mg) prepared by papain cleavage of Rituximab IgG was treated with 30 $\mu \mathrm{g}$ of recombinant S. pyogenes EndoS [22] for $1 \mathrm{hr}$ at $37^{\circ} \mathrm{C}$. Analysis by LC-MS showed complete cleavage of the glycan. The deglycosylated Fc was isolated using a Sephacryl S-200 HR size exclusion column (GE Healthcare) while monitoring UV absorbance and collecting peaks. The fractions containing deglycosylated Fc were pooled and concentrated to give $9 \mathrm{mg}$ of the intermediate (Fuca1,6)GlcNAc-Fc. A solution of (Fuca1,6)GlcNAc-Fc (5 mg) and sialoglycan-oxazoline $(5 \mathrm{mg})$ buffered with Tris- $\mathrm{HCl}$ $(100 \mathrm{mM}, \mathrm{pH} 7.0,0.5 \mathrm{~mL})$ was incubated with EndoS-D233A $(200 \mu \mathrm{g})$ [22] at $30^{\circ} \mathrm{C}$. Aliquots were taken at time intervals for LC-MS analysis of reaction progression. Quantitative conversion was achieved in 2 hours. The product was purified using size exclusion chromatography as described above. Product fractions were pooled and concentrated to give di-sFc $(4.5 \mathrm{mg})$. LC-MS data: calculated for (Fuca1,6)GlcNAc-Fc monomer, $\mathrm{MW}=25287.3 \mathrm{Da}$; found MW = $25289 \mathrm{Da}$ (deconvolution data obtained from the original spectrum). Calculated for fully sialylated N-glycan-Fc monomer, MW = 27288.9 Da; found MW = 27289 Da (deconvolution data).

\section{N-glycan analysis}

$25 \mu \mathrm{g}$ of each Fc fragment was treated with PNGase F (NEB) in a G7 buffer $(0.5$ M sodium phosphate, $\mathrm{pH}$ 7.5) following the manufacturer's instructions. Samples were purified over a CarboPac PGC column and analyzed using HPAEC-PAD on a Dionex ICS 5000 with a CarboPac PA200 column. The $\mathrm{N}$-glycans were eluted in $100 \mathrm{mM} \mathrm{NaOH}$ with the following gradient conditions: $0-10 \mathrm{mM} \mathrm{NaOAC}$ in $20 \mathrm{~min}$ and then $10-100 \mathrm{mM}$ $\mathrm{NaOAc}$ in 20 min. Peaks were assigned using $N$-glycan standards purified from PNGase F digestions of Rituximab, sheep IgG (Sigma), and sialylglycopeptide (SGP) from chicken egg yolk. 


\section{Monosaccharide compositional analysis of putative O-glycans}

PNGase F-treated Fc proteins lacking $N$-glycans were dissolved in 3M TFA in screw cap vials. The tubes were sealed and incubated at $100{ }^{\circ} \mathrm{C}$ for 4 hours. Tubes were then cooled to $25^{\circ} \mathrm{C}$, and the reaction mixture was evaporated to dryness in a SpeedVac. The dried samples were dissolved in water and subjected to HPAEC-PAD analysis on a CarboPac PA10 column. Monosaccharides were eluted with $18 \mathrm{mM} \mathrm{NaOH}$ and detected with pulsed amperic detection with a gold electrode using a standard monosaccharide waveform. For analysis of sialic acids from putative O-glycans, the de$N$-glycosylated Fc proteins were treated with neuraminidase (NEB) for 16 hours in the desialylation buffer provided by the manufacturer (NEB). The samples were then subjected to HPAEC-PAD analysis on a CarboPac PA10 column using a sialic acid standard (Sigma) for detection and quantification.

\section{LC-ESI-MS Analysis of Fc fragments}

Fc fragments and PNGase F-treated Fc fragments were reduced with 50 mM TCEP at $37^{\circ} \mathrm{C}$ for 15 minutes and subjected to LC-ESI-MS analysis on an Agilent C8 column eluted with a gradient of $0-60 \%$ acetonitrile in $10 \mathrm{~min}$.

\section{Crystallization and data collection}

Crystals of di-sFc (space group P2 $2_{1} ; a=52.73 \AA, b=154.22 \AA, c=66.12 \AA, \beta=$ $110.78^{\circ}$; two Fc dimers per asymmetric unit) were grown in sitting drop vapor diffusion by mixing equal volumes of di-sFc $(6.15 \mathrm{mg} / \mathrm{ml})$ with a solution containing $0.2 \mathrm{M}$ magnesium chloride, $0.1 \mathrm{M}$ sodium acetate $\mathrm{pH} 5$, and $20 \%(\mathrm{w} / \mathrm{v})$ PEG 6000 at $20^{\circ} \mathrm{C}$. Crystals were cryopreserved in well solution supplemented with $30 \%$ glycerol. Data were 
collected to 3.0 A resolution at beamline 8.2.1 of the Advanced Light Source (ALS) at Lawrence Berkeley National Laboratory (LBNL).

Crystals of wtFc (space group P $2{ }_{1} 2_{1} 2_{1} ; a=49.56 \AA, b=79.69 \AA, c=138.06 \AA$;

one Fc dimer per asymmetric unit) were grown in sitting drop vapor diffusion by mixing equal volumes of protein with a solution containing $0.1 \mathrm{M}$ sodium cacodylate $\mathrm{pH} 6.5$ and $18 \%(w / v)$ PEG 3350 at $20^{\circ} \mathrm{C}$. The protein solution contained mainly $21 \mathrm{c}$ Fab produced by papain digestion of 21c IgG, but contaminating amounts of the Fc crystallized. The Fc crystals were cryopreserved in well solution containing $30 \%$ glycerol. F241A Fc crystals (space group $\mathrm{P} 2{ }_{1} 2{ }_{1} 2 ; ; a=57.91 \AA, b=74.70 \AA, c=113.71 \AA$; one Fc dimer per asymmetric unit) were grown in hanging drops by mixing F241A Fc $(4.4 \mathrm{mg} / \mathrm{ml})$ with $30 \%$ PEG 1500 at $20^{\circ} \mathrm{C}$ and cryopreserved in well solution containing $25 \%$ glycerol. Data were collected for both wtFc (2.4 Å resolution) and F241A Fc (2.2 A resolution) at beamline 12-2 of the Stanford Synchrotron Radiation Lightsource (SSRL).

\section{Data Processing and Structure Determination}

Diffraction data were processed and indexed using iMosflm [34] and integrated and scaled using POINTLESS and SCALA [35; 36]. In addition to considering $I / \sigma \mathrm{I}$ and completeness of the highest resolution shell, we used the $\mathrm{CC}_{1 / 2}$ statistic [37] (correlation coefficient between two random halves of the data set where $\mathrm{CC}_{1 / 2}>10 \%$ ) to determine the high-resolution cutoff for our data. We used Phenix [38] to compute $\mathrm{CC}_{1 / 2}$ values. Structures were solved by molecular replacement using PHASER [39] and published Fc structures as search models (PDB 1H3X for F241A Fc and wtFc; 3D03 for di-sFc). Modeling was done using COOT [40] using composite omit maps for building glycans and nearby protein residues in the $\mathrm{C}_{\mathrm{H}} 2$ domains. Crystallographic refinement was done using the Phenix crystallography package [38] by refining individual B factors for the higher resolution structures (F241A Fc and wtFc; $2.2 \AA$ and $2.4 \AA$ resolution, 
respectively). For the lower resolution di-sFc structure (3.0 A resolution), we refined group B factors and used non-crystallographic symmetry (NCS) restraints during refinement. Because the $\mathrm{C}_{\mathrm{H}} 2$ domain orientations differed between the two di-sFc molecules in the asymmetric unit, the NCS restraints were applied to individual domains $\left(\mathrm{C}_{\mathrm{H}} 2\right.$ domain residues Phe240 - lle336, and residues $\mathrm{C}_{\mathrm{H}} 3$ domain Pro343 - Leu443 on each Fc chain). We used PyMol [41] for superposition calculations and molecular representations.

The F241A Fc model $\left(R_{\text {free }}=23.2 \% ; R_{\text {work }}=20.6 \%\right)$ included 421 protein residues in the F241A Fc dimer (Pro230 - Leu443 on chain A and Gly237- Leu443 on chain B), 16 glycan residues (GlcNAc1-Gal6, Fuc12 on chain A and GlcNAc1-GlcNAc8, Fuc12 on Chain B), and 351 water molecules. No electron density was observed for residues Thr225 and Cys226 of the Fc hinge or for Man7-NeuNAc10 or Gal9-NeuNAc11 in the $N$-linked glycan of chain A or B, respectively. The wtFc model $\left(R_{\text {free }}=26.4 \% ; R_{\text {work }}\right.$ $=21.1 \%$ ) included 415 protein residues in the wtFc dimer (Pro238-Ser444 on chain $\mathrm{A}$ and Gly236-Pro443 on chain B), 13 glycan residues (GlcNAc1-GlcNAc5, Man7, GlcNAc8, Fuc12 on both chains A and B), and 165 water molecules. No electron density was observed for residues Cys226 to Pro230 of the Fc hinge or for Gal6, Gal9 or NeuNAc10 on the $N$-glycan. Side chains were disordered for residues 269 and 311 on chain A and 246, 269, 311, and 326 on chain B. The di-sFc structure $\left(R_{\text {free }}=28.0 \%\right.$; $R_{\text {work }}=26.0 \%$ ) included two conformationally distinct Fc dimers (chains $A$ and $B$ in the closed dimer and chains $C$ and $D$ in the open dimer). The closed di-sFc dimer model included 414 protein residues (Gly236 - Ser444 and Pro238 - Ser444 on chains A and B, respectively) and 16 glycan residues (GlcNAc1- GlcNAc5, Man7, GlcNAc8 on chain A and GlcNAc1 - GlcNAc8, NeuNAc11 on chain B). No electron density was observed for residues Glu225 to Pro230 of the Fc hinge or for Gal6, Gal9 - NeuNAc10 (chain A) or Gal9, NeuNAc11 on (chain B) in the $N$-linked glycan. Side chains were disordered for 
residues 246, 251, 258, 268, 269, 272, 274, 280, 288, 292, 294, 295, 296, 301, 307 , $317,326,340,355,360,365,386,410,414,416,418$, and 433 on chain $A$ and 248 , $269,272,274,283,298,314,326,340,355,384$, and 386 on chain B. The open di-sFc dimer model included 410 protein residues (residues Pro238 - Leu443 on each Fc chain) and 17 glycan residues (GlcNAc1-GlcNAc8, NeuNAc11 on chain C and,GlcNAc1GlcNAc8 on chain D). No electron density was observed for residues Cys226 to Pro230 of the Fc hinge, Ala327-Ile332 (chain C), Val264-Asp270 (Chain D) or for Gal9, NeuNAc10 (chain C) or Gal9-NeuNAc11 (chain D) in the N-linked glycan. Side chains were disordered for residues 242, 246, 268, 269, 270, 272, 274, 285, 288, 290, 293, $295,300,301,309,311,312,320,340,342,360,380,384,386,416,418,419$, and 433 on chain $\mathrm{C}$ and $248,263,272,276,286,288,290,295,301,302,323,332,334,360$, $377,384,386$, and 439 on chain D.

\section{ACCESSION NUMBERS}

Atomic coordinates and structure factors have been deposited in the Protein Data Bank with accession codes 4Q7D (wtFc), 4Q6Y (di-sFc), and 4Q74 (F241A Fc).

\section{ACKNOWLEDGEMENTS}

We thank Jost Vielmetter and the Caltech Protein Expression Center for protein production, Beth Stadtmueller and members of the Bjorkman, Ravetch, and Wang laboratories for critical reading of the manuscript, Marta Murphy for help making figures, and Jens Kaiser and members of the staff at the Stanford Synchrotron Radiation Lightsource (SSRL) and the Advanced Light Source (ALS) for help with data collection and processing. Operations at SSRL are supported by the US Department of Energy and the National Institutes of Health. The Advanced Light Source is supported by the Director, Office of Science, Office of Basic Energy Sciences, of the U.S. Department of 
Energy under Contract No. DE-AC02-05CH11231. This research was supported by the National Institute Of Allergy And Infectious Diseases of the National Institutes of Health Grant HIVRAD P01 Al100148 (P.J.B. and J.V.R.); (the content is solely the responsibility of the authors and does not necessarily represent the official views of the National Institutes of Health), the National Institutes of Health (R01 Al041239 to P.J.B.), Award DP1OD006961 from the Office of The Director, National Institutes of Health (to P.J.B.), R01 GM096973 (to L.X.W.), R56 Al034662 (to J.V.R.), and the Molecular Observatory at Caltech supported by the Gordon and Betty Moore Foundation. P.J.B. is a Howard Hughes Medical Institute investigator. 


\section{REFERENCES}

1. Nimmerjahn, F. \& Ravetch, J. V. (2008). Anti-inflammatory actions of intravenous immunoglobulin. Annu Rev Immunol 26, 513-33.

2. Anthony, R. M. \& Ravetch, J. V. (2010). A novel role for the IgG Fc glycan: the anti-inflammatory activity of sialylated IgG Fcs. J Clin Immunol 30 Suppl 1, S9-14.

3. Nimmerjahn, F. \& Ravetch, J. V. (2011). FcgammaRs in health and disease. Curr Top Microbiol Immunol 350, 105-25.

4. Samuelsson, A., Towers, T. L. \& Ravetch, J. V. (2001). Anti-inflammatory activity of IVIG mediated through the inhibitory Fc receptor. Science 291, 484-6.

5. Tackenberg, B., Jelcic, I., Baerenwaldt, A., Oertel, W. H., Sommer, N., Nimmerjahn, F. \& Lunemann, J. D. (2009). Impaired inhibitory Fcgamma receptor IIB expression on $B$ cells in chronic inflammatory demyelinating polyneuropathy. Proc Natl Acad Sci U S A 106, 4788-92.

6. Gelfand, E. W. (2006). Differences between IGIV products: impact on clinical outcome. Int Immunopharmacol 6, 592-9.

7. Anthony, R. M., Nimmerjahn, F., Ashline, D. J., Reinhold, V. N., Paulson, J. C. \& Ravetch, J. V. (2008). Recapitulation of IVIG anti-inflammatory activity with a recombinant IgG Fc. Science 320, 373-6.

8. Nimmerjahn, F. \& Ravetch, J. V. (2007). The antiinflammatory activity of IgG: the intravenous IgG paradox. J Exp Med 204, 11-5.

9. Kaneko, Y., Nimmerjahn, F. \& Ravetch, J. V. (2006). Anti-inflammatory activity of immunoglobulin $\mathrm{G}$ resulting from Fc sialylation. Science 313, 670-3.

10. Arnold, J. N., Wormald, M. R., Sim, R. B., Rudd, P. M. \& Dwek, R. A. (2007). The impact of glycosylation on the biological function and structure of human immunoglobulins. Annu Rev Immunol 25, 21-50.

11. Schwab, I., Mihai, S., Seeling, M., Kasperkiewicz, M., Ludwig, R. J. \& Nimmerjahn, F. (2014). Broad requirement for terminal sialic acid residues and FcgammaRIIB for the preventive and therapeutic activity of intravenous immunoglobulins in vivo. Eur $\mathrm{J}$ Immunol.

12. Anthony, R. M., Kobayashi, T., Wermeling, F. \& Ravetch, J. V. (2011). Intravenous gammaglobulin suppresses inflammation through a novel $\mathrm{T}(\mathrm{H}) 2$ pathway. Nature $475,110-3$.

13. Sondermann, P., Pincetic, A., Maamary, J., Lammens, K. \& Ravetch, J. V. (2013). General mechanism for modulating immunoglobulin effector function. Proc Natl Acad Sci U S A 110, 9868-72.

14. Krapp, S., Mimura, Y., Jefferis, R., Huber, R. \& Sondermann, P. (2003). Structural analysis of human IgG-Fc glycoforms reveals a correlation between glycosylation and structural integrity. J Mol Biol 325, 979-89.

15. Feige, M. J., Nath, S., Catharino, S. R., Weinfurtner, D., Steinbacher, S. \& Buchner, J. (2009). Structure of the murine unglycosylated IgG1 Fc fragment. J Mol Biol 391, 599-608.

16. Ferrara, C., Grau, S., Jager, C., Sondermann, P., Brunker, P., Waldhauer, I., Hennig, M., Ruf, A., Rufer, A. C., Stihle, M., Umana, P. \& Benz, J. 
(2011). Unique carbohydrate-carbohydrate interactions are required for high affinity binding between FcgammaRIII and antibodies lacking core fucose. Proc Natl Acad Sci U S A 108, 12669-74.

17. Matsumiya, S., Yamaguchi, Y., Saito, J., Nagano, M., Sasakawa, H., Otaki, S., Satoh, M., Shitara, K. \& Kato, K. (2007). Structural comparison of fucosylated and nonfucosylated Fc fragments of human immunoglobulin G1. J Mol Biol 368, 767-79.

18. Nimmerjahn, F. \& Ravetch, J. V. (2005). Divergent immunoglobulin g subclass activity through selective Fc receptor binding. Science 310 , 1510-2.

19. Shields, R. L., Lai, J., Keck, R., O'Connell, L. Y., Hong, K., Meng, Y. G., Weikert, S. H. \& Presta, L. G. (2002). Lack of fucose on human IgG1 Nlinked oligosaccharide improves binding to human Fcgamma RIII and antibody-dependent cellular toxicity. J Biol Chem 277, 26733-40.

20. Barb, A. W., Meng, L., Gao, Z., Johnson, R. W., Moremen, K. W. \& Prestegard, J. H. (2012). NMR characterization of immunoglobulin G Fc glycan motion on enzymatic sialylation. Biochemistry 51, 4618-26.

21. Sprague, E. R., Martin, W. L. \& Bjorkman, P. J. (2004). pH dependence and stoichiometry of binding to the Fc region of $\mathrm{IgG}$ by the herpes simplex virus Fc receptor gE-gl. J Biol Chem 279, 14184-93.

22. Huang, W., Giddens, J., Fan, S. Q., Toonstra, C. \& Wang, L. X. (2012). Chemoenzymatic glycoengineering of intact lgG antibodies for gain of functions. J Am Chem Soc 134, 12308-18.

23. Yu, X., Baruah, K., Harvey, D. J., Vasiljevic, S., Alonzi, D. S., Song, B. D., Higgins, M. K., Bowden, T. A., Scanlan, C. N. \& Crispin, M. (2013).

Engineering hydrophobic protein-carbohydrate interactions to fine-tune monoclonal antibodies. J Am Chem Soc 135, 9723-32.

24. Shibuya, N., Goldstein, I. J., Broekaert, W. F., Nsimba-Lubaki, M., Peeters, B. \& Peumans, W. J. (1987). The elderberry (Sambucus nigra L.) bark lectin recognizes the Neu5Ac(alpha 2-6)Gal/GalNAc sequence. J Biol Chem 262, 1596-601.

25. Lund, J., Takahashi, N., Pound, J. D., Goodall, M. \& Jefferis, R. (1996). Multiple interactions of $\lg G$ with its core oligosaccharide can modulate recognition by complement and human Fc gamma receptor I and influence the synthesis of its oligosaccharide chains. J Immunol 157, 4963-9.

26. Crispin, M., Bowden, T. A., Coles, C. H., Harlos, K., Aricescu, A. R., Harvey, D. J., Stuart, D. I. \& Jones, E. Y. (2009). Carbohydrate and domain architecture of an immature antibody glycoform exhibiting enhanced effector functions. J Mol Biol 387, 1061-6.

27. Oganesyan, V., Damschroder, M. M., Leach, W., Wu, H. \& Dall'Acqua, W. F. (2008). Structural characterization of a mutated, ADCC-enhanced human Fc fragment. Mol Immunol 45, 1872-82.

28. Oganesyan, V., Damschroder, M. M., Woods, R. M., Cook, K. E., Wu, H. \& Dall'acqua, W. F. (2009). Structural characterization of a human Fc fragment engineered for extended serum half-life. Mol Immunol 46, 17505. 
29. Teplyakov, A., Zhao, Y., Malia, T. J., Obmolova, G. \& Gilliland, G. L. (2013). Ig 2 Fc structure and the dynamic features of the IgG CH2-CH3 interface. Mol Immunol 56, 131-9.

30. Crispin, M., Yu, X. \& Bowden, T. A. (2013). Crystal structure of sialylated IgG Fc: implications for the mechanism of intravenous immunoglobulin therapy. Proc Natl Acad Sci U S A 110, E3544-6.

31. Anthony, R. M., Wermeling, F., Karlsson, M. C. \& Ravetch, J. V. (2008). Identification of a receptor required for the anti-inflammatory activity of IVIG. Proc Natl Acad Sci U S A 105, 19571-8.

32. Frank, M., Walker, R. C., Lanzilotta, W. N., Prestegard, J. H. \& Barb, A. W. (2014). Immunoglobulin G1 Fc Domain Motions: Implications for Fc Engineering. J Mol Biol.

33. Diskin, R., Marcovecchio, P. M. \& Bjorkman, P. J. (2010). Structure of a clade C HIV-1 gp120 bound to CD4 and CD4-induced antibody reveals anti-CD4 polyreactivity. Nat Struct Mol Biol 17, 608-13.

34. Leslie, A. G. W. \& Powell, H. R. (2007). Processing DIffraction Data with Mosfilm. In Evolving Methods for Macromolecular Crystallography, Vol. 245, pp. 41-51.

35. Evans, P. R. (2011). An introduction to data reduction: space-group determination, scaling and intensity statistics. Acta Crystallogr D Biol Crystallogr 67, 282-92.

36. Evans, P. (2006). Scaling and assessment of data quality. Acta Crystallogr D Biol Crystallogr 62, 72-82.

37. Karplus, P. A. \& Diederichs, K. (2012). Linking crystallographic model and data quality. Science 336, 1030-3.

38. Adams, P. D., Afonine, P. V., Bunkoczi, G., Chen, V. B., Davis, I. W., Echols, N., Headd, J. J., Hung, L. W., Kapral, G. J., Grosse-Kunstleve, R. W., McCoy, A. J., Moriarty, N. W., Oeffner, R., Read, R. J., Richardson, D. C., Richardson, J. S., Terwilliger, T. C. \& Zwart, P. H. (2010). PHENIX: a comprehensive Python-based system for macromolecular structure solution. Acta Crystallogr D Biol Crystallogr 66, 213-21.

39. McCoy, A. J., Grosse-Kunstleve, R. W., Adams, P. D., Winn, M. D., Storoni, L. C. \& Read, R. J. (2007). Phaser crystallographic software. J Appl Crystallogr 40, 658-674.

40. Emsley, P., Lohkamp, B., Scott, W. G. \& Cowtan, K. (2010). Features and development of Coot. Acta Crystallogr D Biol Crystallogr 66, 486-501.

41. Schrödinger, L. (2011). The PyMOL Molecular Graphics System 1.2r3pre edit. The PyMOL Molecular Graphics System. 
Table 1: Distance measurements between $\mathrm{C}_{\mathrm{H}} 2$ domains in IgG1 Fc structures

\begin{tabular}{|c|c|c|c|c|c|c|c|c|c|c|}
\hline $\begin{array}{l}\text { PDB } \\
\text { ID }\end{array}$ & Protein & Category & $\begin{array}{c}\text { How } \\
\text { expressed }\end{array}$ & $\begin{array}{l}\text { Space } \\
\text { Group }\end{array}$ & $\begin{array}{c}\text { Cell } \\
\text { Dimensions } \\
\text { a,b,c }(\AA) \\
\end{array}$ & $\begin{array}{c}\text { Resolution } \\
(\AA)\end{array}$ & $\begin{array}{c}\text { Pro238 } \\
\mathrm{Ca}(\AA)\end{array}$ & $\begin{array}{c}\text { Phe241 } \\
\text { Ca }(\AA)\end{array}$ & $\begin{array}{l}\operatorname{Arg} 301 \\
\operatorname{Ca}(\AA)\end{array}$ & $\begin{array}{l}\text { Pro } 329 \\
\text { Ca }(\AA)\end{array}$ \\
\hline $1 \mathrm{H} 3 \mathrm{X}$ & $\mathrm{Fc}(\mathrm{G} 0 \mathrm{~F}) 2$ & wtFc & $\lg G$ & $P 22_{1} 2_{1}$ & $50,80,139$ & 2.4 & 19.7 & 21.9 & 33.9 & 22.6 \\
\hline 3AVE & Fc Fucosylated & wtFc & $\lg G$ & $P 22_{1} 2_{1}$ & $50,78,144$ & 2.0 & 19.3 & 21.8 & 32.3 & 25.1 \\
\hline 2DTS & Fc Defucosylated & wtFc & $\lg G$ & $P 2_{1} 2_{1} 2_{1}$ & $49,78,143$ & 2.2 & 18.6 & 21.5 & 32.2 & 24.2 \\
\hline $3 \mathrm{DO} 3$ & $\mathrm{Fc}$ & wtFc & $\lg G$ & $P 2_{1} 2_{1} 2_{1}$ & $50,80,139$ & 2.5 & 20.1 & 22.4 & 32.8 & 23.5 \\
\hline 1FC1 & Fc & wtFc & $\lg G$ & $P 2_{1} 2_{1} 2_{1}$ & $80,146,50$ & 2.9 & 19.6 & 23.5 & 34.2 & 26.8 \\
\hline N/A & $\mathrm{Fc}$ & wtFc & $\lg G$ & $P 2_{1} 2_{1} 2_{1}$ & $50,80,138$ & 2.4 & 20.2 & 21.9 & 32.9 & 22.9 \\
\hline $1 \mathrm{HZH}$ & Intact IgG1 & wtFc & $\lg G$ & H32 & $271,271,175$ & 2.7 & 21.1 & 22.2 & 33.6 & 23.9 \\
\hline $1 \mathrm{H} 3 \mathrm{Y}$ & Fc high salt & $\begin{array}{l}\text { wtFc high } \\
\text { salt }\end{array}$ & $\lg G$ & $P 6_{1} 22$ & $153,153,117$ & 4.1 & 16.7 & 22.3 & 25.9 & 29.6 \\
\hline $4 \mathrm{KU} 1$ & Fc $(\mathrm{G} 2 \mathrm{~F}) 2$ & glycovariant & $\lg G$ & $P 2{ }_{1} 2_{1} 2_{1}$ & $49,80,128$ & 1.9 & 21.3 & 23.3 & 33.2 & 29.1 \\
\hline $1 \mathrm{H} 3 \mathrm{~V}$ & Fc (G2F)2 & glycovariant & $\lg G$ & $P 2,22_{1}$ & $49,81,142$ & 3.1 & 19.3 & 23.2 & 32.9 & 26.9 \\
\hline $1 \mathrm{H} 3 \mathrm{~W}$ & $\mathrm{Fc}(\mathrm{G} 2 \mathrm{~F}) 2$ & glycovariant & $\lg G$ & $C 222_{1}$ & $50,149,76$ & 2.9 & 22.9 & 26.1 & 35.9 & 33.8 \\
\hline $1 \mathrm{H} 3 \mathrm{U}$ & $\mathrm{Fc}(\mathrm{M} 3 \mathrm{~N} 2 \mathrm{~F}) 2$ & glycovariant & $\lg G$ & $P 2{ }_{1} 2_{1} 2_{1}$ & $49,80,144$ & 2.4 & 19.2 & 22.0 & 32.7 & 24.2 \\
\hline $2 \mathrm{WAH}$ & Fc (Man9NAG2) & glycovariant & $\mathrm{Fc}$ & $P 2_{1} 2_{1} 2_{1}$ & $49,75,149$ & 2.5 & 30.2 & 29.8 & 42.8 & 36.4 \\
\hline $4 \mathrm{ACP}$ & $\mathrm{Fc}(\mathrm{GlcNAc})$ & glycovariant & $\mathrm{Fc}$ & C2 & $\begin{array}{c}69,111,78, \\
\beta=108^{\circ}\end{array}$ & 2.5 & 11.4 & 17.8 & 26.2 & $\mathrm{~N} / \mathrm{A}$ \\
\hline 3DNK & Fc deglycosylated & glycovariant & $\lg G$ & $P 2_{1} 2_{1} 2_{1}$ & $49,78,147$ & 2.8 & 24.8 & 25.0 & 37.4 & 27.6 \\
\hline $3 S 7 G$ & Fc deglycosylated & glycovariant & $?$ & $P 1$ & $66,66,78$ & 3.1 & 10.7 & 16.8 & 25.8 & 18.9 \\
\hline 2QL1 & Fc DLE mutant & mutant Fc & Fc & $C 222_{1}$ & $50,147,74$ & 2.5 & 27.6 & 28.5 & 37.2 & 39.3 \\
\hline 3FJT & Fc YTE mutant & mutant Fc & Fc & $P 2_{1} 2_{1} 2_{1}$ & $50,80,146$ & 2.5 & 20.0 & 22.1 & 33.4 & 25.3 \\
\hline $3 \mathrm{C} 2 \mathrm{~S}$ & Fc FES mutant & mutant Fc & $\lg G$ & $C 222_{1}$ & $50,147,75$ & 2.3 & 28.1 & 28.8 & 38.1 & 35.9 \\
\hline $3 D 6 G$ & Fc: protein A & complex & $\lg G$ & $P 2_{1} 2_{1} 2_{1}$ & $50,79,139$ & 2.3 & 19.2 & 22.2 & 32.9 & 24.3 \\
\hline 1L6X & Fc: Z34C & complex & $?$ & $C 222_{1}$ & $54,125,90$ & 1.7 & 22.2 & 22.8 & 33.9 & 26.6 \\
\hline 10QO & Fc (G0): mini Z & complex & $?$ & $P 2,2,2$ & $86,126,55$ & 2.3 & 20.0 & 22.1 & 33.1 & 24.3 \\
\hline $10 Q X$ & Fc (G2): Z34C & complex & $?$ & $P 2,2,2$ & $87,126,54$ & 2.6 & 20.1 & 21.8 & 33.0 & 24.7 \\
\hline 1DN2 & Fc: peptide & complex & $\lg G$ & $P 2_{1}$ & $\begin{array}{c}68,61,68 \\
\beta=104^{\circ}\end{array}$ & 2.7 & 26.1 & 27.0 & 37.4 & 31.9 \\
\hline $1 \mathrm{FCC}$ & Fc: Protein G & complex & $\lg G$ & $P 4_{3} 2_{1} 2$ & $111,111,160$ & 3.2 & 22.1 & 26.0 & 34.5 & 34.7 \\
\hline $1 \mathrm{FC} 2$ & Fc: protein $A$ & complex & $\lg G$ & $P 3,21$ & $71,72,147$ & 2.8 & 18.3 & 22.0 & 31.4 & 27.7 \\
\hline $1 \mathrm{E} 4 \mathrm{~K}$ & Fc :FcgRIIIB & complex & $\lg G$ & $P 6_{5} 22$ & $115,115,299$ & 3.2 & 25.0 & 25.7 & 36.7 & 30.3 \\
\hline $1 \mathrm{~T} 83$ & Fc :FcgRIIIB & complex & $\lg G$ & $P 2_{1} 2_{1} 2_{1}$ & $73,102,123$ & 3.0 & 24.8 & 26.1 & 36.9 & 31.3 \\
\hline $1 T 89$ & Fc :FcgRIIIB & complex & $\lg G$ & $P 6_{5} 22$ & $115,115,301$ & 3.5 & 24.5 & 25.9 & 37.3 & 30.6 \\
\hline $3 A Y 4$ & $\begin{array}{c}\text { Fc afucosylated: } \\
\text { FcgRIIIA }\end{array}$ & complex & $\lg G$ & $P 4{ }_{1}{ }_{1} 2$ & $77,77,350$ & 2.2 & 25.2 & 25.7 & 36.3 & 30.8 \\
\hline $3 R Y 6$ & Fc: FcgRIla & complex & $\lg G$ & $C 222_{1}$ & $153,256,58$ & 3.8 & 20.5 & 23.1 & 34.4 & 31.4 \\
\hline 3SGK & $\begin{array}{c}\text { afucosylated Fc: } \\
\text { FcyRIIIA }\end{array}$ & complex & $\lg G$ & $P 2{ }_{1} 2_{1} 2_{1}$ & $67,88,141$ & 2.4 & 25.6 & 26.6 & 37.4 & 32.6 \\
\hline 3SGJ & $\begin{array}{c}\text { fucosylated Fc: } \\
\text { FcyRIIIA }\end{array}$ & complex & $\lg G$ & $P 2{ }_{1} 2_{1} 2_{1}$ & $67,88,140$ & 2.2 & 25.6 & 26.3 & 36.9 & 32.3 \\
\hline $\mathrm{N} / \mathrm{A}$ & di-sFc closed & $\mathrm{N} / \mathrm{A}$ & $\lg G$ & $P 2_{1}$ & $\begin{array}{c}53,154,66, \\
\beta=111^{\circ}\end{array}$ & 3.2 & 12.8 & 17.8 & 26.4 & 21.9 \\
\hline N/A & di-sFc open & $\mathrm{N} / \mathrm{A}$ & $\lg G$ & $P 2_{1}$ & $\begin{array}{c}53,154,66 \\
\beta=111^{\circ}\end{array}$ & 3.2 & 20.1 & 21.7 & 31.2 & N/A \\
\hline 4BYH & $\mathrm{sFc}$ & $\mathrm{N} / \mathrm{A}$ & $?$ & $P 6,22$ & $153,153,112$ & 2.3 & 17.6 & 21.8 & 26.2 & 30.5 \\
\hline $\mathrm{N} / \mathrm{A}$ & F241A Fc & $\mathrm{N} / \mathrm{A}$ & $\mathrm{Fc}$ & $P 2,2{ }_{1} 2_{1}$ & $58,75,114$ & 2.2 & 23 & 25.1 & 33.8 & 31.1 \\
\hline 4BM7 & F241A Fc & $\mathrm{N} / \mathrm{A}$ & Fc & $P 2_{1} 2_{1} 2_{1}$ & $49,73,136$ & 1.9 & 34 & 31.7 & 45.1 & $\mathrm{~N} / \mathrm{A}$ \\
\hline
\end{tabular}


Figure 1

(a)

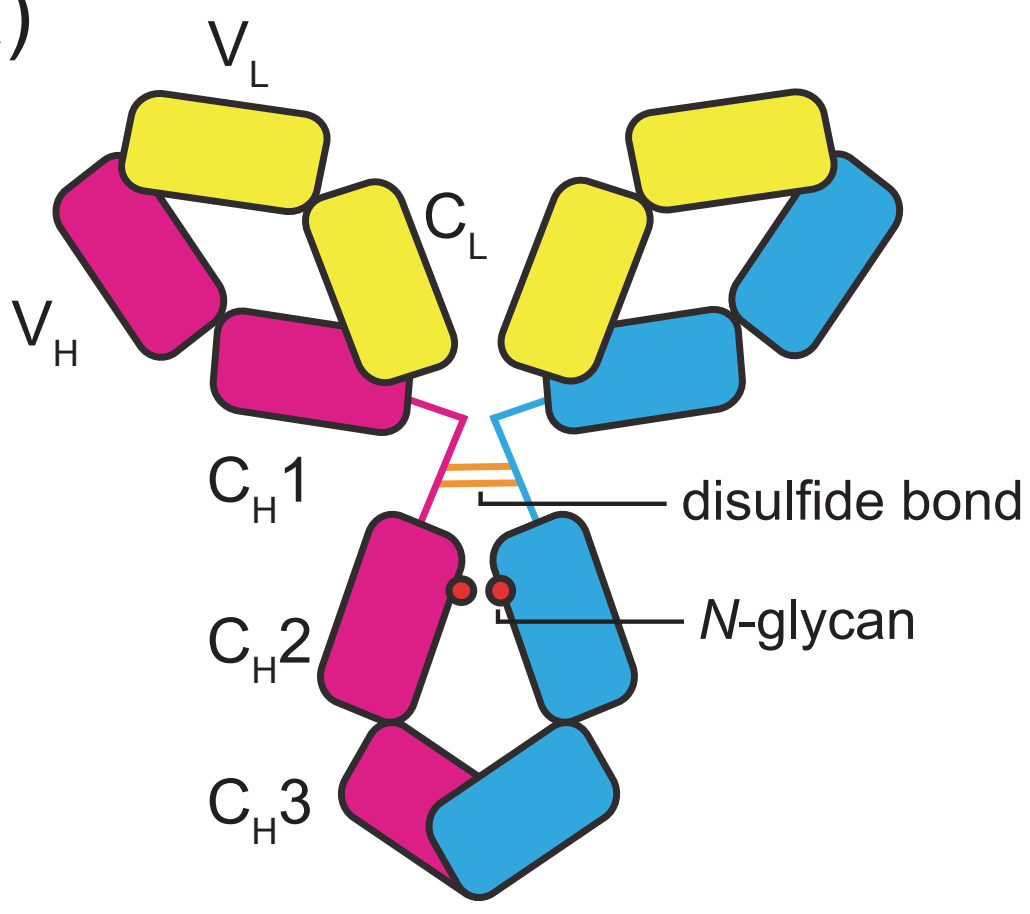

(b) Chain A Chain B

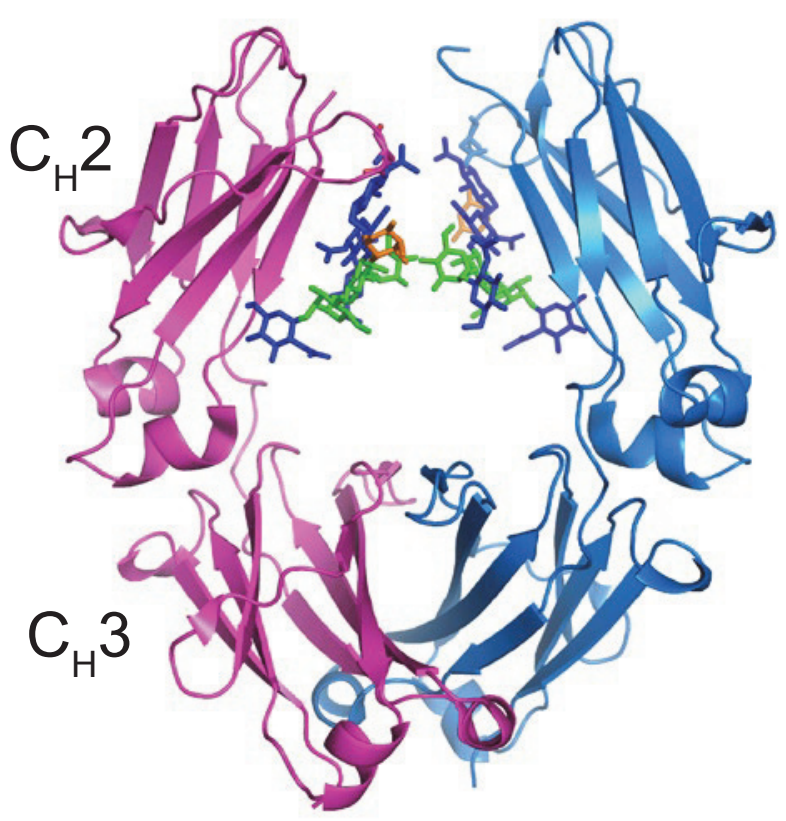

(c)

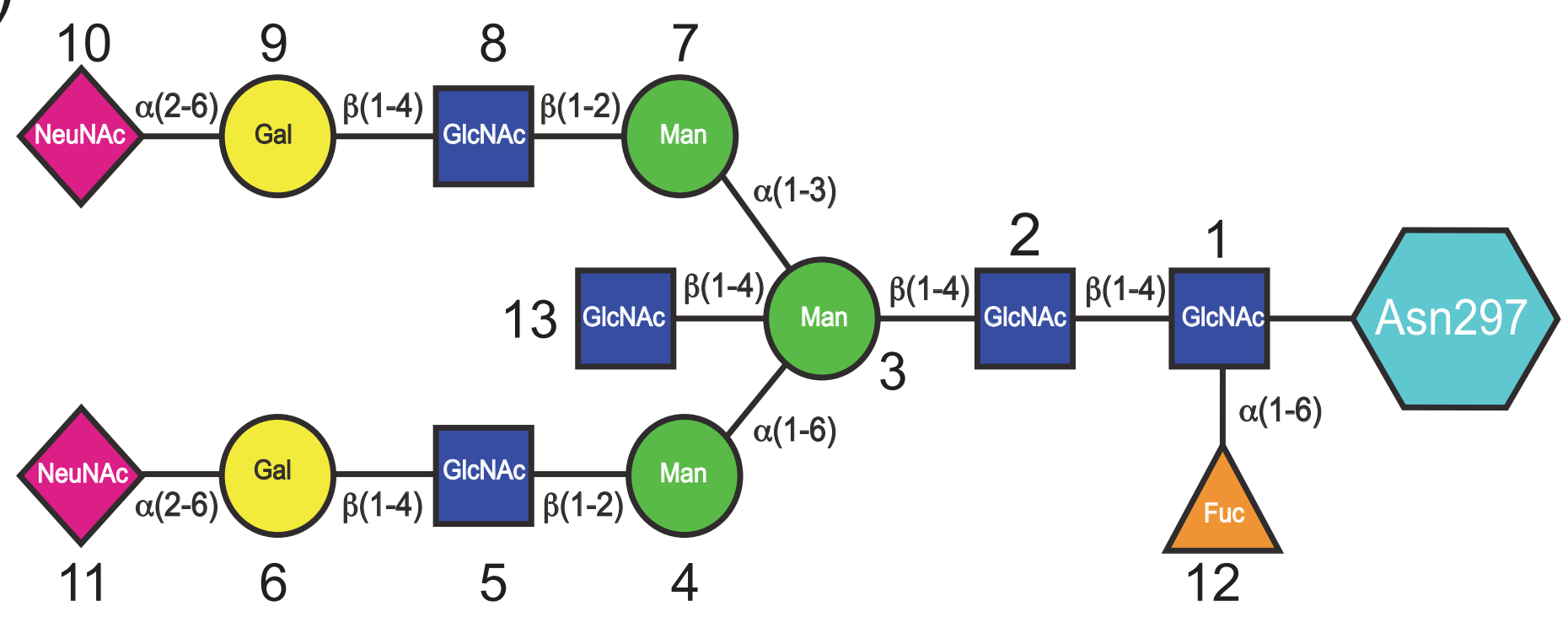

Fig. 1 
(a)

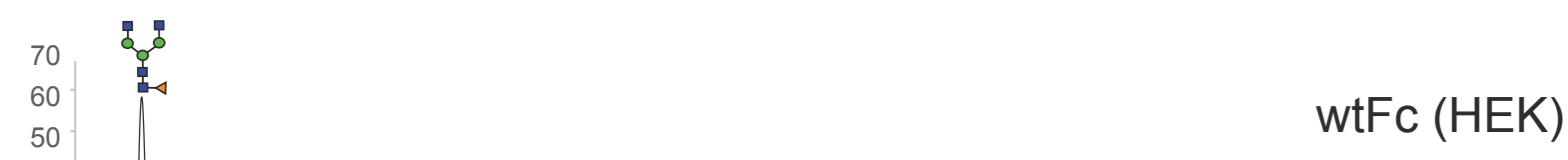

(b)

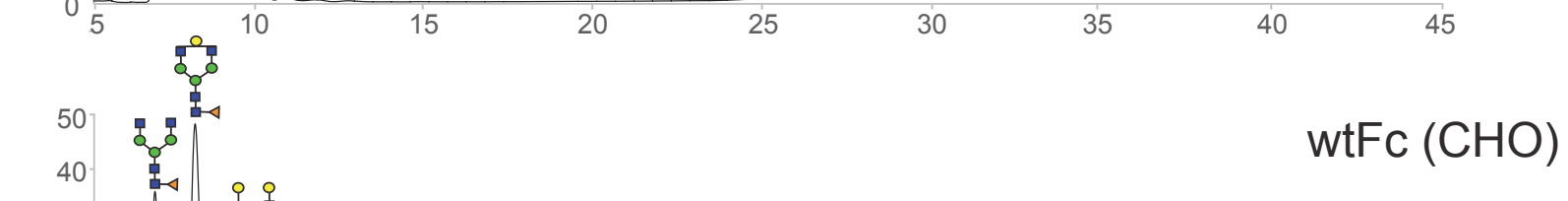

$0 \% \mathrm{sFc}$

$0 \% \mathrm{sFc}$

(c)

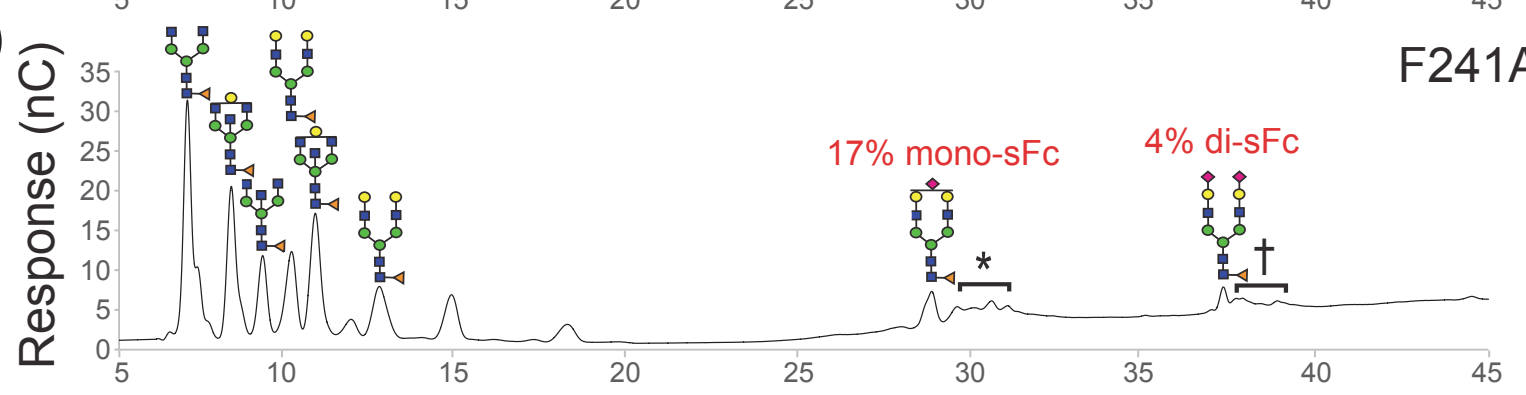

(d)

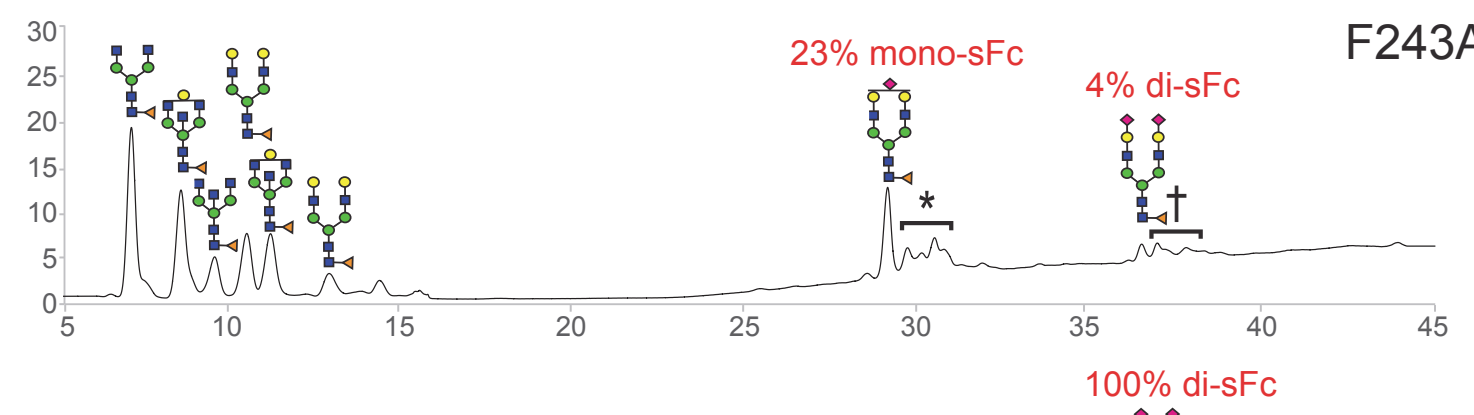

(e)
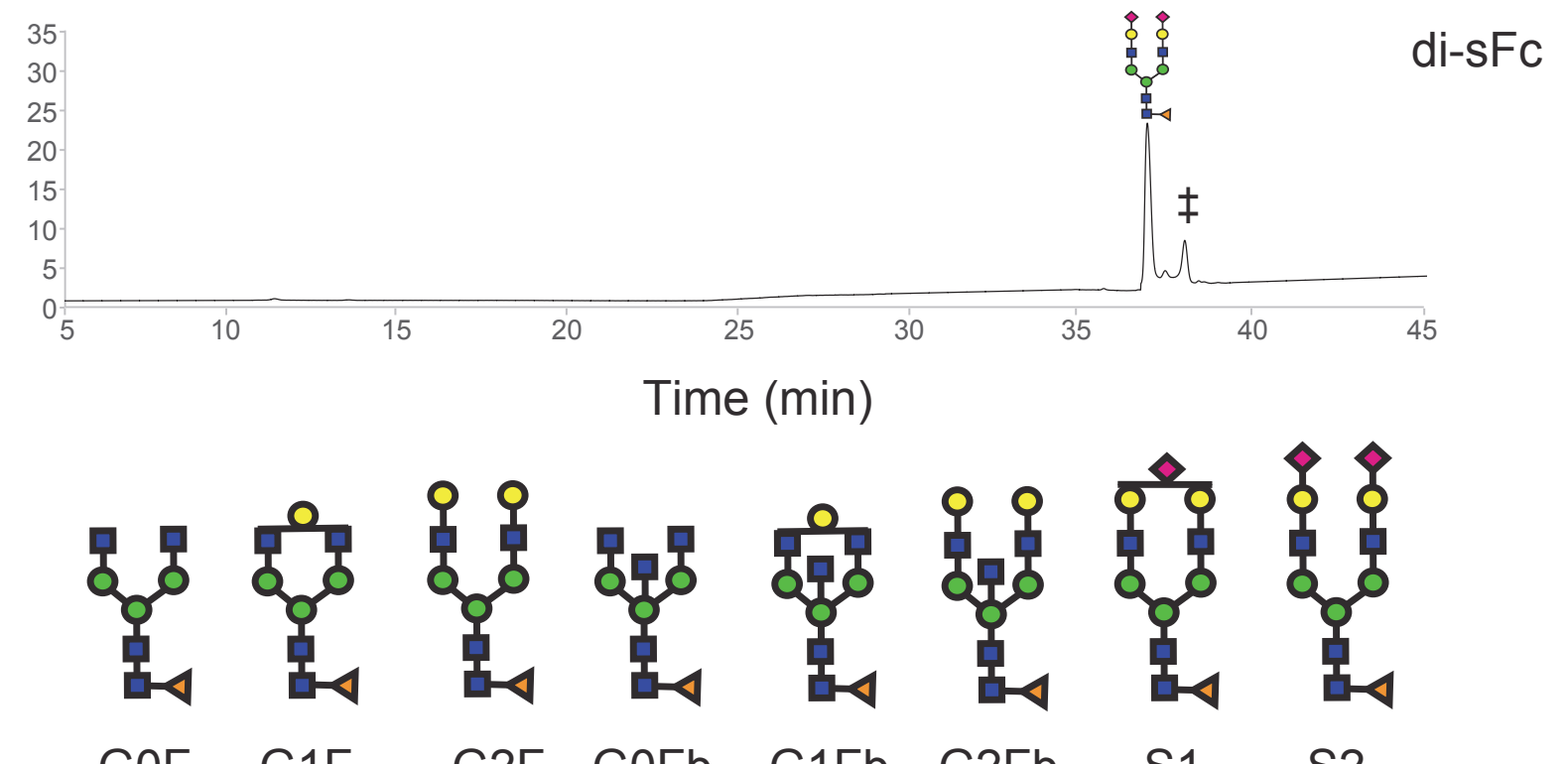

G0F G1F G2F G0Fb G1Fb G2Fb S1 S2

( $\square$ GIcNAc; O Man; O Gal; $\triangleleft$ Fuc; $\diamond$ Sia)

Fig. 2 
Figure 3

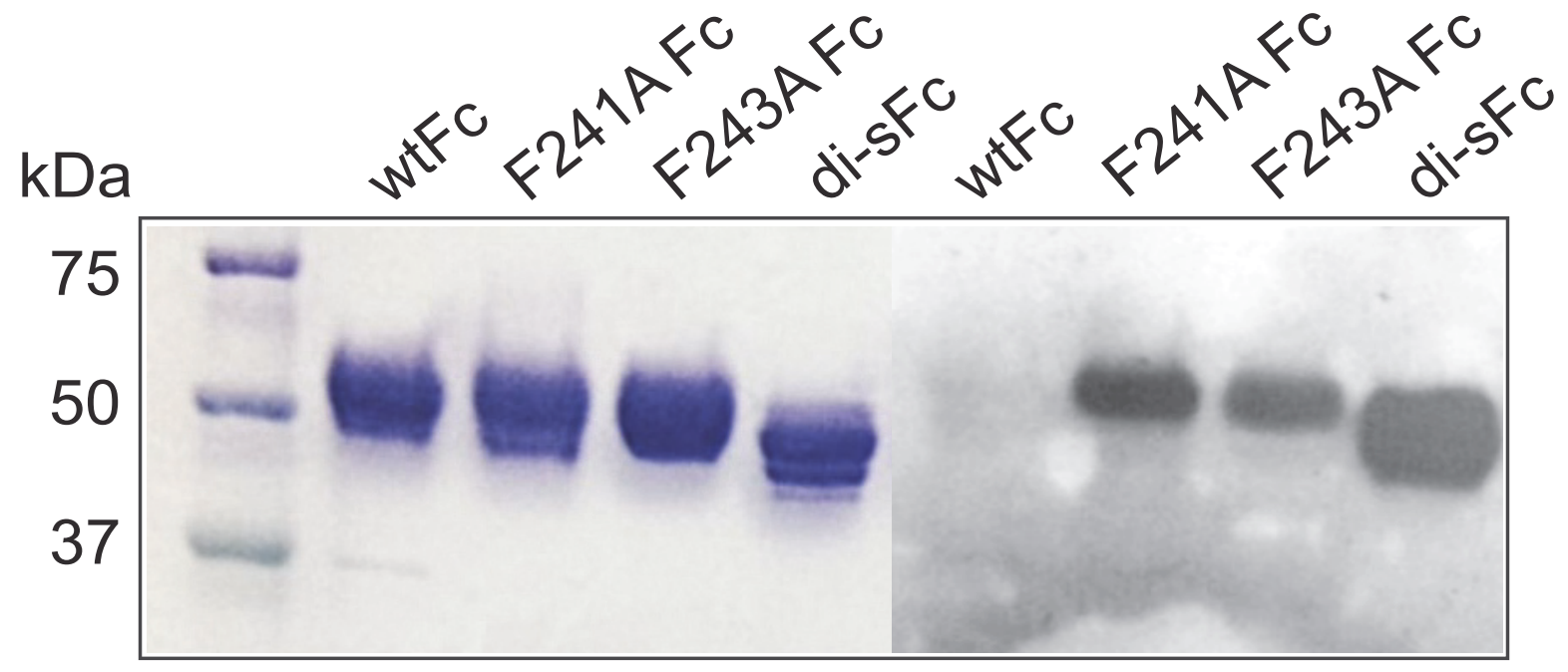

Fig. 3 
(a)

(b)

Chain A

Chain B

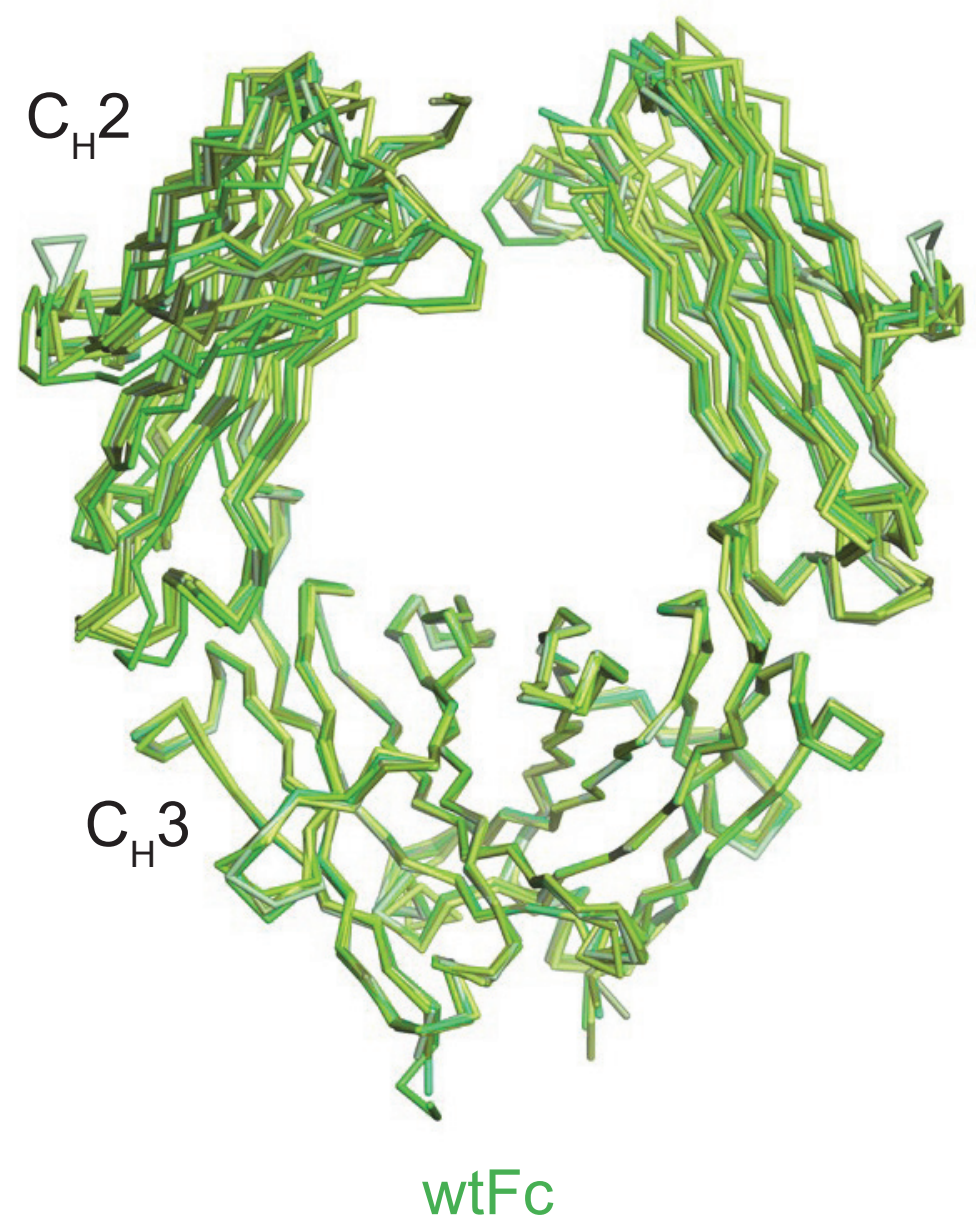

Chain A

Chain B

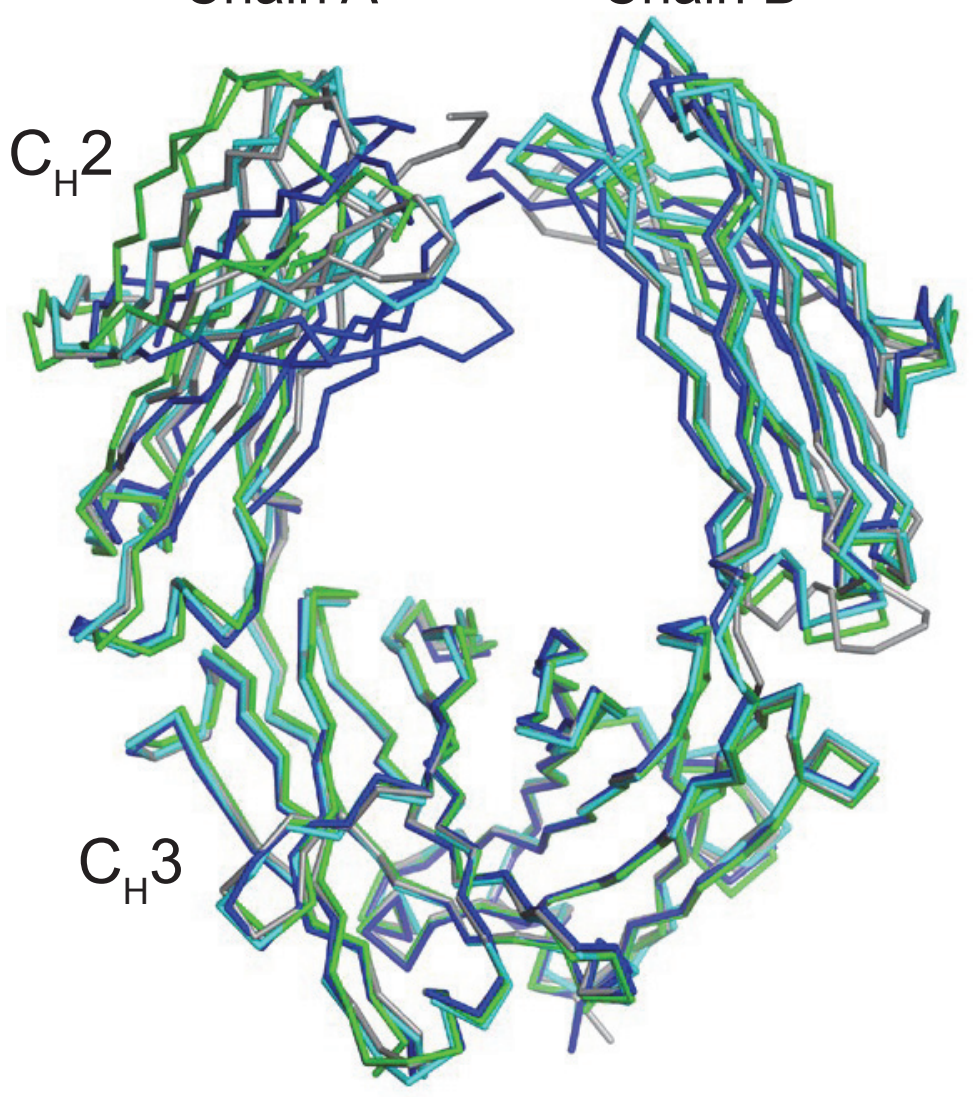

wtFc (3AVE), sFc (4BYH)
di-sFc (closed), di-sFc (open)

Fig. 4 


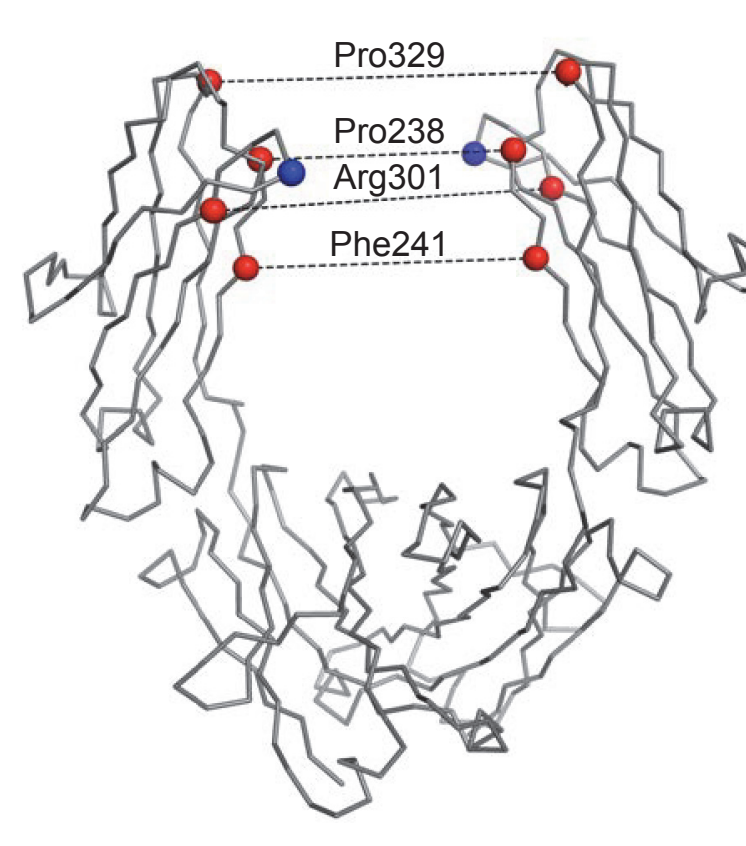

Pro238

Phe241
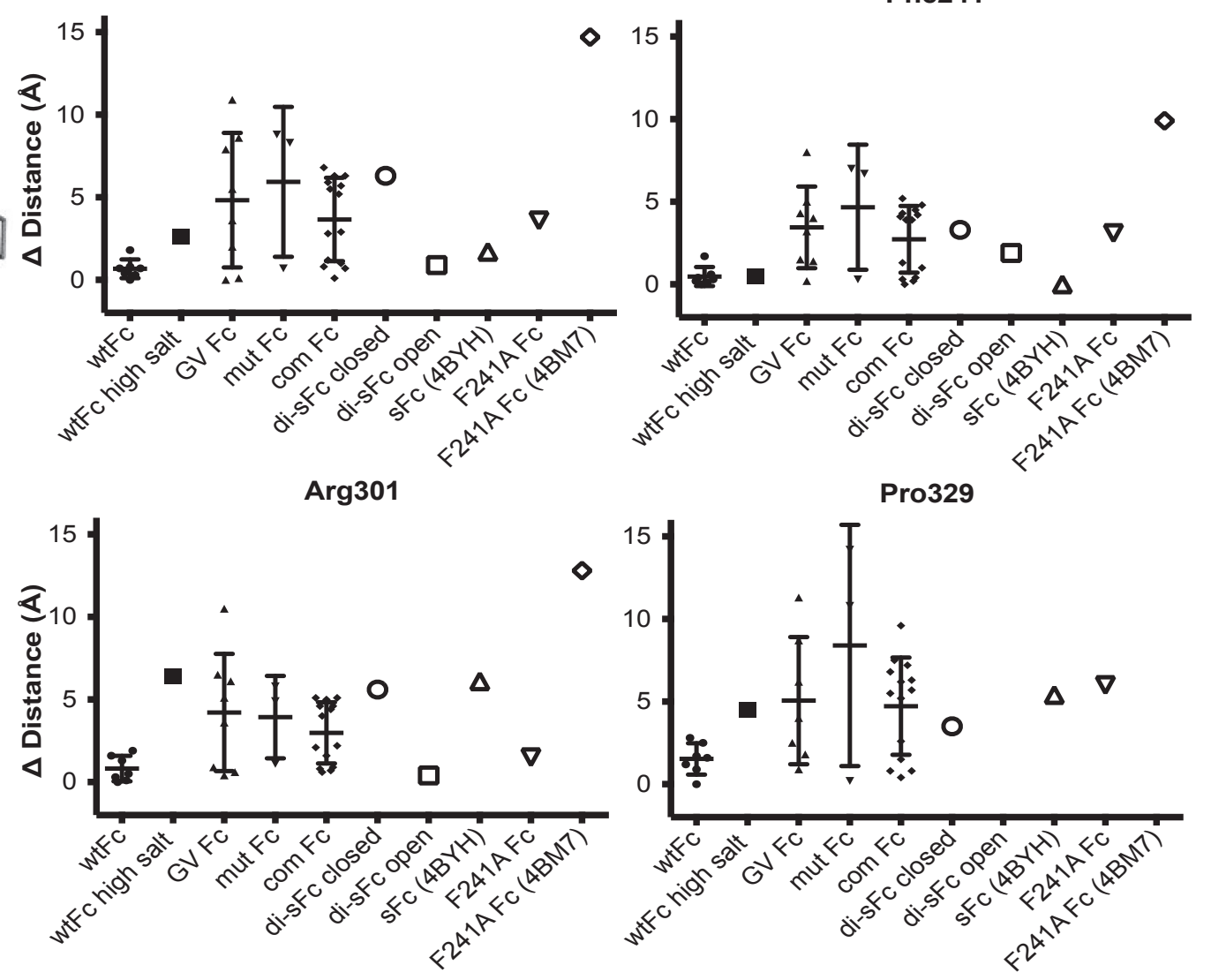
Figure 7
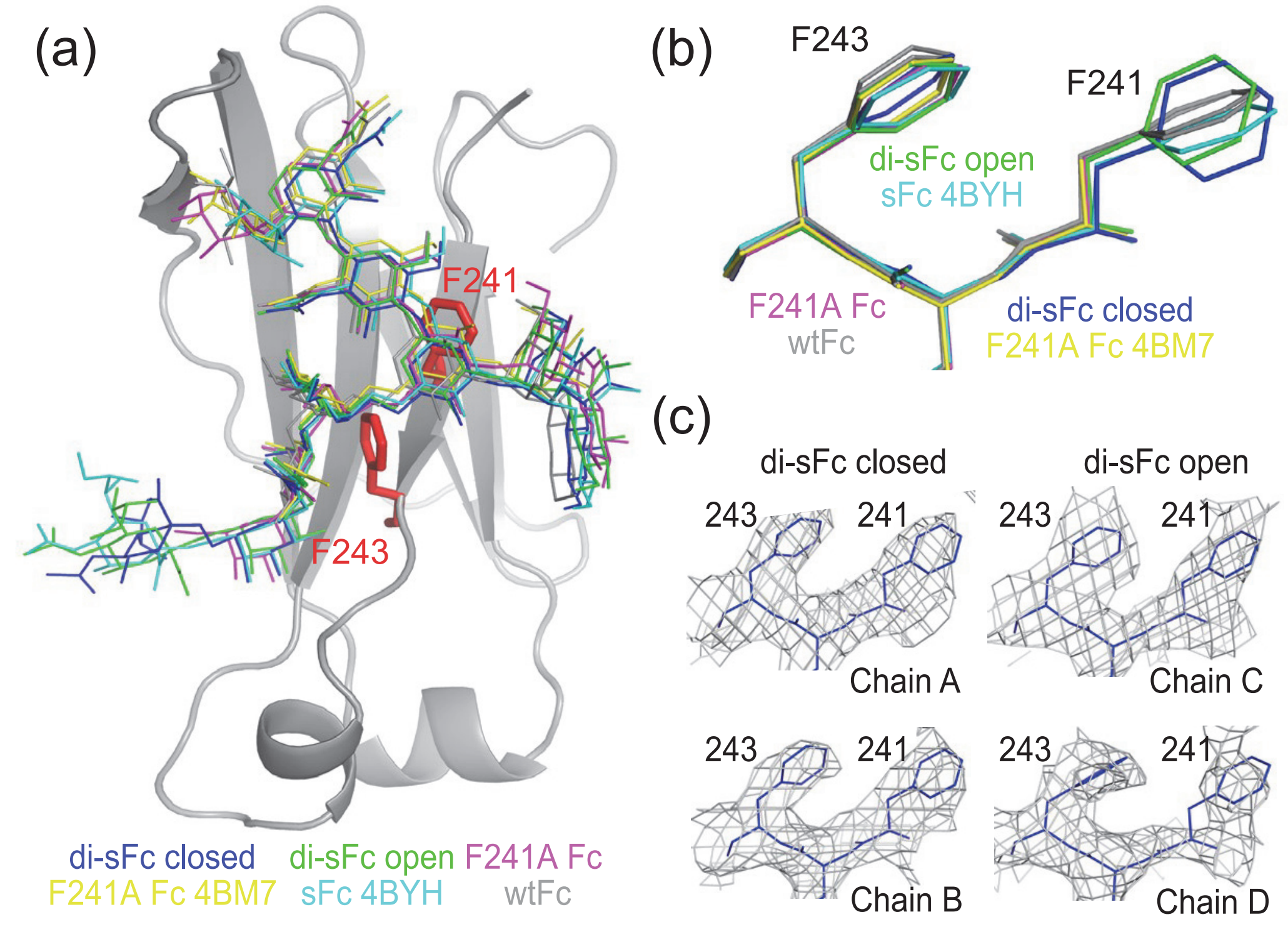

Fig. 7 
(a)

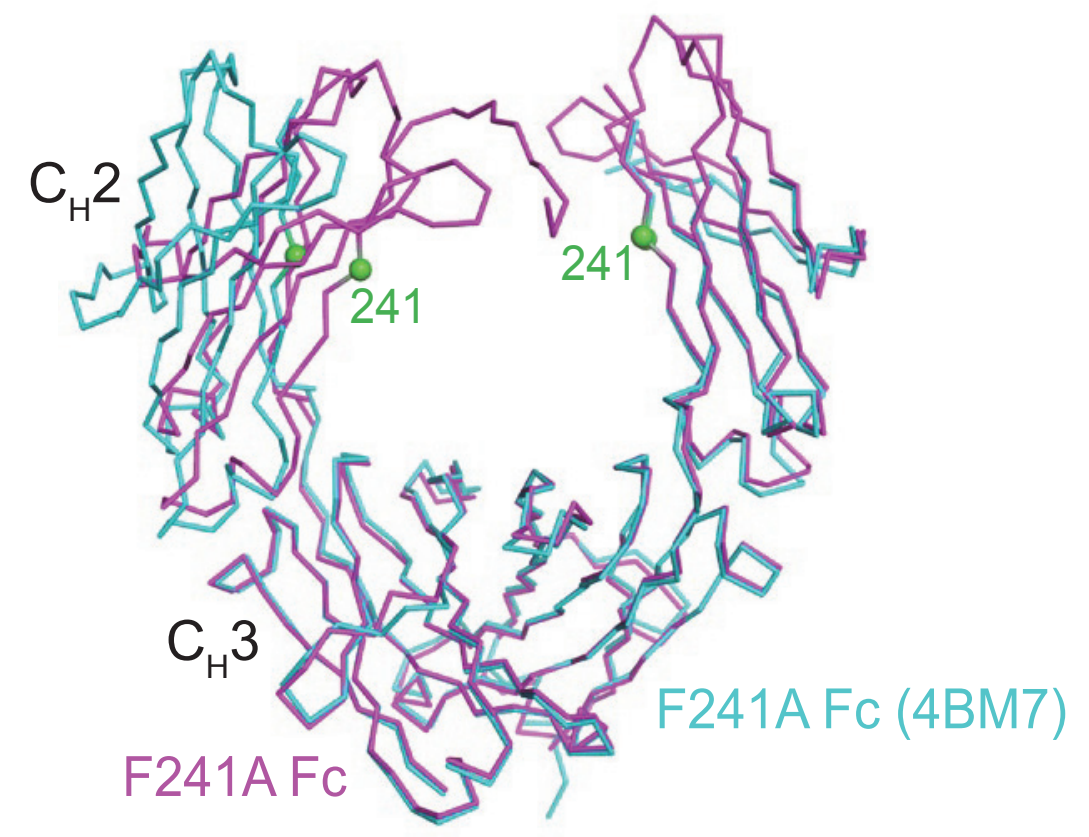

(c)

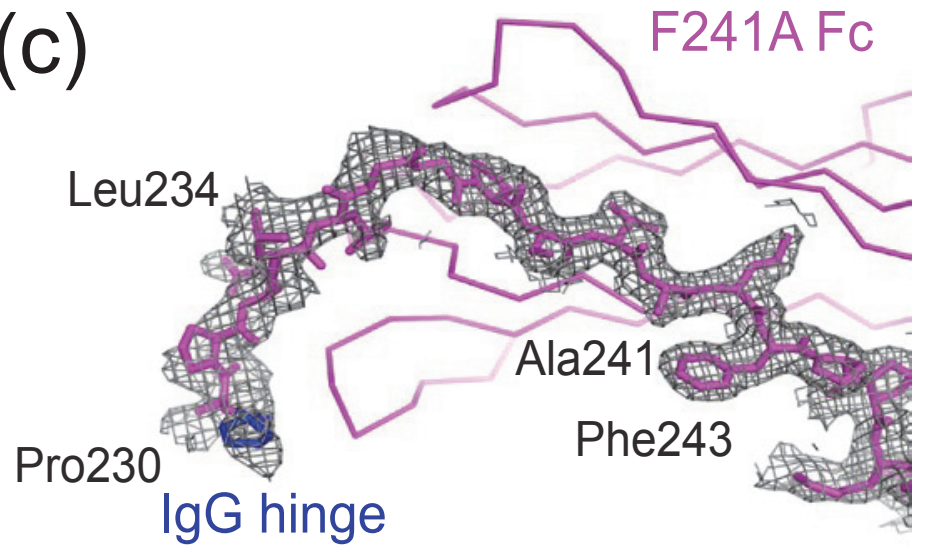

(d) (b)

F241A Fc

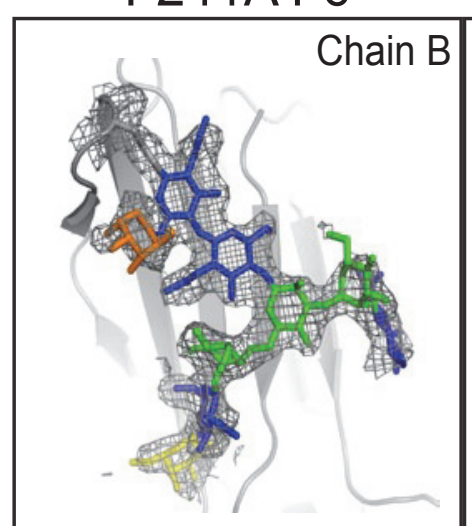

3-arm

6-arm O-g-O Chain A

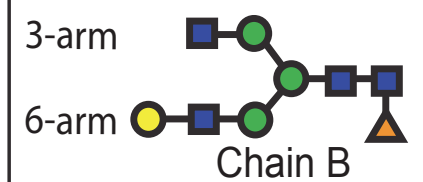

F241A Fc (4BM7) Chain A
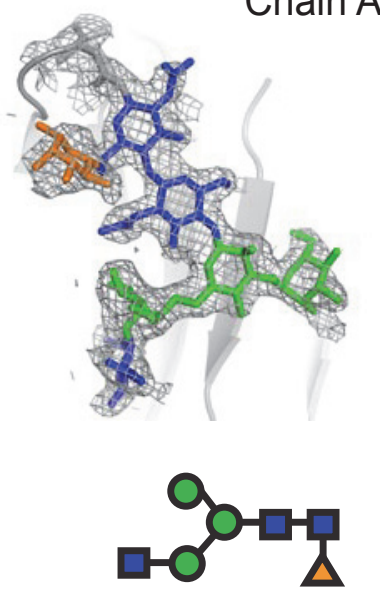

Chain A

Chain B disordered

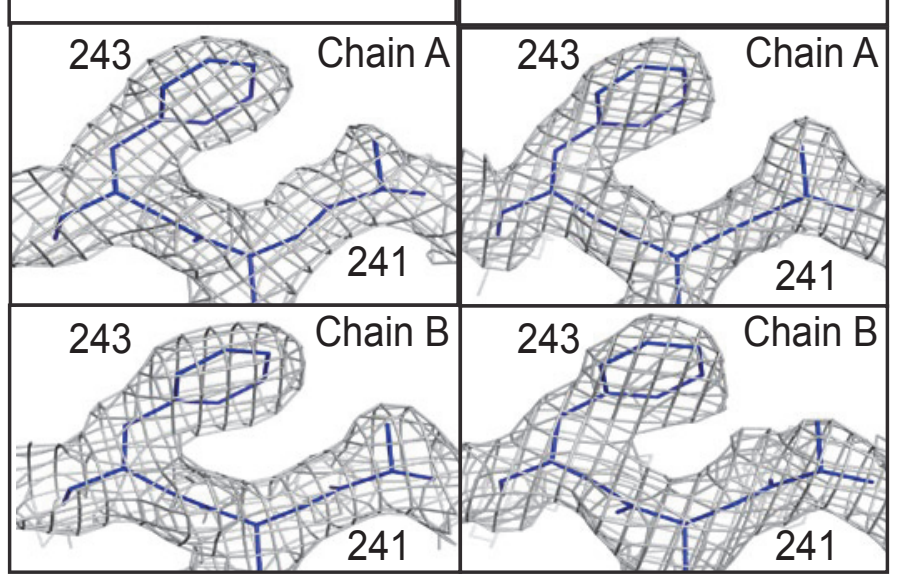

Fig. 8 

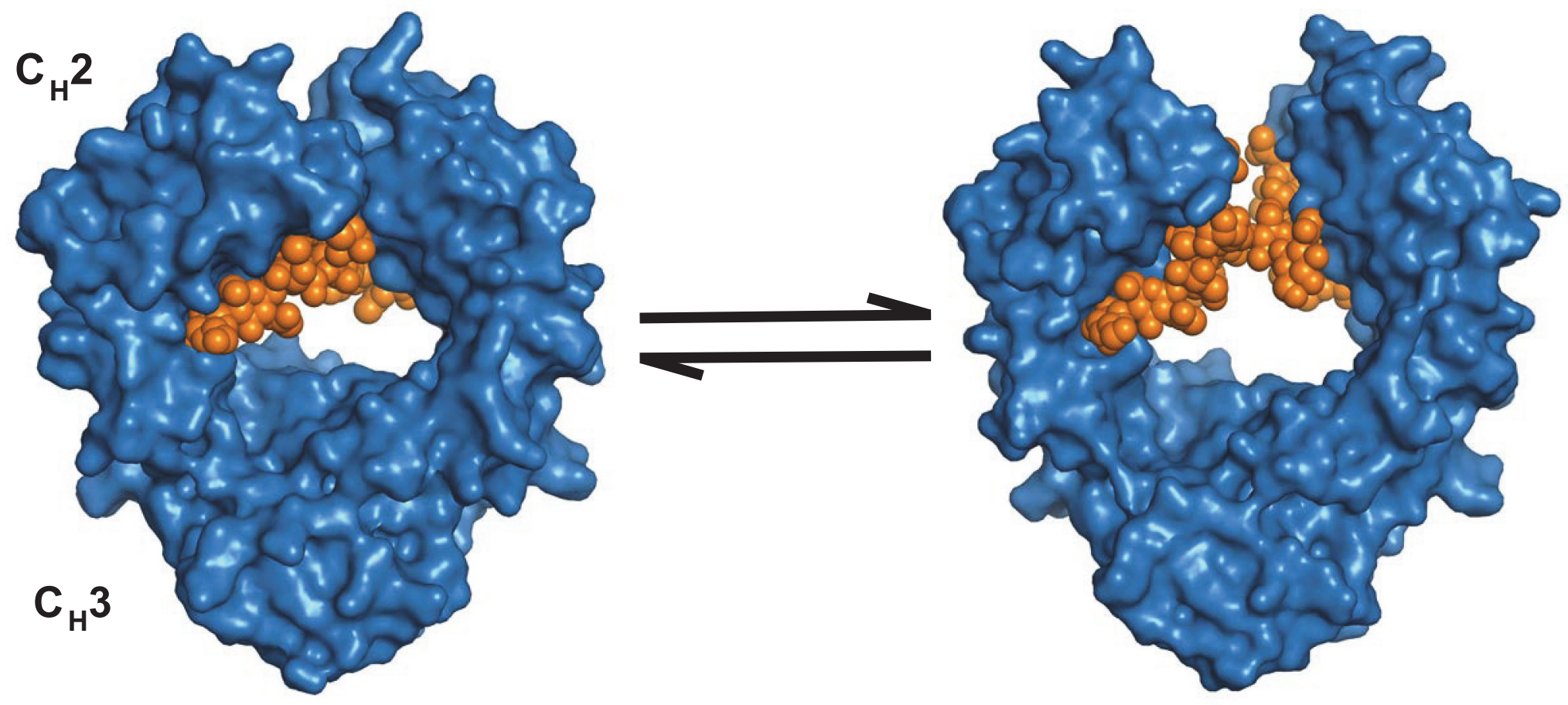

Increased conformational flexibility of IgG Fc associated with switch from pro- to anti-inflammatory activity 